\title{
HIGHER ORDER HILBERT-SCHMIDT HANKEL FORMS AND TENSORS OF ANALYTIC KERNELS
}

\author{
SARAH H. FERGUSON and RICHARD ROCHBERG*
}

\begin{abstract}
The symbols of $n^{\text {th }}$-order Hankel forms defined on the product of certain reproducing kernel Hilbert spaces $H\left(k_{i}\right), i=1,2$, in the Hilbert-Schmidt class are shown to coincide with the orthogonal complement in $H\left(k_{1}\right) \otimes H\left(k_{2}\right)$ of the ideal of polynomials which vanish up to order $n$ along the diagonal. For tensor products of weighted Bergman and Dirichlet type spaces (including the Hardy space) we introduce a higher order restriction map which allows us to identify the relative quotient of the $n^{\text {th }}$-order ideal modulo the $(n+1)^{\text {st }}$-order one as a direct sum of single variable Bergman and Dirichlet-type spaces. This generalizes the well understood $0^{\text {th }}$-order case.
\end{abstract}

\section{Overview}

\subsection{Contents}

Let $H^{2}\left(\mathrm{D}^{2}\right)$ be the Hardy space of the bidisk, the Hilbert space of functions $f(z, w)=\sum_{n, m \geq 0} a_{n m} z^{n} w^{m}$ with $\sum\left|a_{n m}\right|^{2}<\infty$. Sitting inside $H^{2}\left(\mathbf{D}^{2}\right)$ is $V_{D}$, the subspace of functions which vanish on the diagonal $D=\{(z, w)$ : $z=w\} \cap \mathrm{D}^{2}$. When studying the function theory of $V_{D}$ and its orthogonal complement $V_{D}^{\perp}$ one finds that the restriction map, $r$, defined by $(r f)(\zeta)=$ $f(\zeta, \zeta)$ is a partial isometry of $H^{2}\left(\mathrm{D}^{2}\right)$ to a Hilbert space of functions on $D$, that the reproducing kernel of that function space can be described explicitly, and that $P=r^{*} r$ is the orthogonal projection $H^{2}\left(\mathrm{D}^{2}\right)$ onto $V_{D}^{\perp}$. Furthermore there is an explicit formula for this projection

$$
P f(z, w)=\frac{1}{z-w} \int_{w}^{z} f(\zeta, \zeta) d \zeta .
$$

These facts, which are quite classical, can easily be verified using reproducing kernels.

In this paper we extend this analysis further in several ways. We obtain analogous results for the relative quotients $V_{D}^{n} \ominus V_{D}^{n+1}$, here $V_{D}^{n}$ is the subspace of $H^{2}\left(\mathrm{D}^{2}\right)$ of functions vanishing on $D$ to order $n$; the results just described are the case $n=0$. We will identify this relative quotient isometrically with

\footnotetext{
* Both authors were supported in part by grants from the National Science Foundation.

Received June 12, 2003.
} 
$Q_{n}$, a Hilbert space of functions on $D$, we will identify the reproducing kernel of $Q_{n}$, and we will give a partial isometry of $H^{2}\left(\mathrm{D}^{2}\right)$ to $Q_{n}$ having initial space $V_{D}^{n} \ominus V_{D}^{n+1}$. This partial isometry is a "higher order" restriction operator obtained by composing $r$ with a certain differential operator. This will allow us to give an explicit form for the orthogonal projection of $H^{2}\left(\mathrm{D}^{2}\right)$ to $V_{D}^{n} \ominus V_{D}^{n+1}$. We will also see that we have an intertwining of the compression of the operator of multiplication by (either) coordinate function to $V_{D}^{n} \ominus V_{D}^{n+1}$ and the operator of multiplication by the coordinate functions on $Q_{n}$. All this is in Section 3 .

Our interest in $H^{2}\left(\mathrm{D}^{2}\right)$ arose because it is a natural realization of the Hilbert space of Hilbert-Schmidt bilinear forms on the one variable Hardy space $H^{2}$ (D). In our analysis we make systematic use of the facts that $H^{2}(\mathrm{D})$ is a Hilbert space with reproducing kernel, that the kernel functions have a simple and explicit form, and that the monomials are orthogonal. Hence it is not surprising that the analysis can be extended beyond the Hardy space to various Bergman and Dirichlet spaces of holomorphic functions on the disk and on the polydisk which share certain basic properties. We will present the analysis at that level of generality; however we emphasize that the core ideas are already present in the case of $H^{2}(\mathrm{D})$.

We then investigate what happens when the Hardy space of the disk is replaced with the Hardy space of the ball. Our approach in that context is quite computational and hence we only consider the ball in $\mathrm{C}^{2}$. New difficulties arise in that context and although we make some progress our results are not as complete.

In the final section we mention some questions.

\subsection{Hankel Forms}

Our interest in these questions arose when studying higher order Hankel forms. We now take a quick look at that topic to see how it is related to the work here. However the work here is independent of considerations of Hankel forms.

Higher order Hankel forms were introduced by Janson and Peetre in [9] and [10] as classes of bilinear forms acting on Möbius invariant function spaces on the disk. They were described in a natural way, namely as the irreducible components of the induced action of the Möbius group on the space bilinear forms. Briefly speaking, Janson and Peetre showed that to each irreducible component of a fixed weight corresponds a class of forms, the so-called special Hankel forms of corresponding weight. The Hankel forms of weight 0 are the usual Hankel forms. By exploiting the action of the infinitesimal operators on kernel functions, the authors found an explicit integral representation of the symbol functions of the special Hankel forms of higher weight and using this realization established conditions on the symbols for boundedness and Schatten-von Neumann properties of the forms. 
In the space of Hilbert-Schmidt bilinear forms the special Hankel forms of different weights are orthogonal. Thus the orthogonal decomposition of Hilbert-Schmidt forms as sums of special Hankel forms of various weights induces, via the identification of the form with its symbol, an orthogonal direct sum decomposition of a space of functions on the bidisk. This connection between the analytic function theory on the bidisk and the symbols of special Hankel forms of higher weight was not made explicit in [9] nor, in [23], but rather in [14].

Motivated by the observation that the symbols of the usual Hankel forms only depend on the restriction of the function to the diagonal $D$, Peetre and Rochberg in [14] defined Hankel forms of higher order by considering the higher order Taylor behavior of the symbols along the diagonal. For the case of weighted Bergman spaces on the disk, they showed that the two notions of generalized Hankel forms yield the same class of forms of order 1 (or, weight 2). The definition of higher order Hankel forms given in [14] has a natural generalization to the multi-variable setting and we follow that approach here and define higher order forms by their commutation properties with multiplication by the coordinate function. In Section 2 this is seen to be equivalent to the definition in terms of Taylor data on the diagonal. Although we do not use the group action in our definition we will see in Section 3 that, for all orders, our approach yields the same class of forms as those obtained using group actions in [9] and [23]. That is, for the case of weighted Bergman spaces on the polydisk, the special higher order Hankel forms we obtain, i.e., forms which are orthogonal to forms of lower order, do coincide with the irreducible components of the action of the automorphism group of the polydisk on bounded bilinear forms. This is Corollary 3.4. It is not clear if this holds for the spaces of functions on the ball.

We would like to mention an alternative generalization to higher dimensions. Rosengren, in [18], extended the results in [9], [10] and [24] by identifying the irreducible components of the d-fold tensor of the action of the Möbius group acting on the d-fold Hilbert space tensor product of weighted Bergman spaces. The corresponding forms are the so-called multi-linear Hankel forms of higher order. The higher order Hankel forms that are studied in this paper are bilinear forms.

\section{Preliminaries}

For $i=1,2$ let $k_{i}$ be an analytic reproducing kernel on a domain $\mathscr{G} \subseteq \mathrm{C}^{d}$ and let $H\left(k_{i}\right)$ denote the Hilbert space of holomorphic functions on $\mathscr{G}$ generated by $k_{i}$. We assume throughout that the operator $M_{z_{j}}$, multiplication by the $j^{\text {th }}$ coordinate function $z_{j}$, is bounded on $H\left(k_{i}\right), i=1,2, j=1, \ldots, d$, and 
that the polynomials, $\mathrm{C}\left[z_{1}, \ldots, z_{d}\right]$, are dense in $H\left(k_{i}\right), i=1,2$. There is a canonical identification (cf. [21]) between the Hilbert space tensor product $H\left(k_{1}\right) \otimes_{2} H\left(k_{2}\right)$ and the Hilbert space of holomorphic functions on $\mathscr{G} \times \mathscr{G}$ with reproducing kernel

$$
\left(k_{1} \otimes k_{2}\right)\left(z_{1}, w_{1}, z_{2}, w_{2}\right)=k_{1}\left(z_{1}, z_{2}\right) k_{2}\left(w_{1}, w_{2}\right) .
$$

We will denote this latter space by $H\left(k_{1} \otimes k_{2}\right)$.

The $2 d$-tuple of multiplication operators on $H\left(k_{1} \otimes k_{2}\right)$ will be denoted by $\left(\mathbf{M}_{z}, \mathbf{M}_{w}\right)$ where $\mathbf{M}_{z}=\left(M_{z_{1}} \otimes I_{H\left(k_{2}\right)}, \ldots, M_{z_{d}} \otimes I_{H\left(k_{2}\right)}\right)$ and $\mathbf{M}_{w}=\left(I_{H\left(k_{1}\right)} \otimes\right.$ $\left.M_{w_{1}}, \ldots, I_{H\left(k_{1}\right)} \otimes M_{w_{d}}\right)$. For a multi-index $I=\left(i_{1}, \ldots, i_{d}\right) \in \mathrm{Z}_{+}^{d}$ we define

$$
\left(\mathbf{M}_{z}-\mathbf{M}_{w}\right)^{I}=\left(M_{z_{1}}-M_{w_{1}}\right)^{i_{1}} \ldots\left(M_{z_{d}}-M_{w_{d}}\right)^{i_{d}} .
$$

and similarly,

$$
(z-w)^{I}=\left(z_{1}-w_{1}\right)^{i_{1}} \ldots\left(z_{d}-w_{d}\right)^{i_{d}}
$$

In particular, note that with this notational convention we have, for $z, w \in \mathrm{C}^{d}$ and a positive integer $k$

$$
\langle z, w\rangle^{k}=\sum_{|I|=k} \frac{k !}{I !} z^{I} w^{I}
$$

\subsection{Hilbert-Schmidt forms and the symbol map}

Let Bilin $\left(k_{1}, k_{2}\right)$ denote the space of bounded bilinear forms defined on $H\left(k_{1}\right) \times$ $H\left(k_{2}\right)$. By the symbol of a form $B \in \operatorname{Bilin}\left(k_{1}, k_{2}\right)$ we mean the function $\pi(B)$, holomorphic on $\mathscr{G} \times \mathscr{G}$, defined by

$$
\pi(B)(z, w):=\overline{B\left(k_{1}(\cdot, z), k_{2}(\cdot, w)\right)} .
$$

A form $B \in \operatorname{Bilin}\left(k_{1}, k_{2}\right)$ is in the Hilbert-Schmidt class if and only if its symbol, $\pi(B)$, is in $H\left(k_{1} \otimes k_{2}\right)$. We will let $\operatorname{Bilin}_{H S}\left(k_{1}, k_{2}\right)$ denote the HilbertSchmidt forms on $H\left(k_{1}\right) \times H\left(k_{2}\right)$. The symbol map $\pi: \operatorname{Bilin}_{H S}\left(k_{1}, k_{2}\right) \rightarrow$ $H\left(k_{1} \otimes k_{2}\right)$, is an isometric isomorphism. Indeed, for any function $h \in H\left(k_{1} \otimes\right.$ $k_{2}$ ) we have, by the reproducing property of the kernel, $h(z, w)=\left\langle h, k_{1}(\cdot, z) \otimes\right.$ $\left.k_{2}(\cdot, w)\right\rangle, z, w \in \Omega$. Thus, by (1.2),

$$
\pi(B)(z, w)=\left\langle\pi(B), k_{1}(\cdot, z) \otimes k_{2}(\cdot, w)\right\rangle .
$$

Since the kernel functions $k_{1}(\cdot, z) \otimes k_{2}(\cdot, w)$ span $H\left(k_{1}\right) \otimes H\left(k_{2}\right)$, it follows that

$$
B\left(f_{1}, f_{2}\right)=\left\langle f_{1} \otimes f_{2}, \pi(B)\right\rangle \quad \forall f_{i} \in H\left(k_{i}\right), \quad i=1,2 .
$$




\subsection{Higher order Hankel forms}

For a polynomial $p \in \mathrm{C}\left[z_{1}, \ldots, z_{d}\right]$ we define the maps $L_{p}$ and $R_{p}$ on $\operatorname{Bilin}\left(k_{1}, k_{2}\right)$ by

$$
L_{p} B\left(f_{1}, f_{2}\right)=B\left(p f_{1}, f_{2}\right) \quad \text { and } \quad R_{p} B\left(f_{1}, f_{2}\right)=B\left(f_{1}, p f_{2}\right) .
$$

For $j=1, \ldots, d$, define $\triangle_{j}=L_{z_{j}}-R_{z_{j}}\left(z_{j}\right.$ denotes the $j^{\text {th }}$ coordinate function). Note that $\triangle_{i} \triangle_{j}=\triangle_{j} \triangle_{i}$ for all $1 \leq i, j \leq d$. For a multi-index of non-negative integers $I=\left(i_{1}, \ldots, i_{d}\right) \in \mathrm{Z}_{+}^{d}$, we define

$$
\triangle^{I}=\triangle_{1}^{i_{1}} \ldots \triangle_{d}^{i_{d}} .
$$

A form $B \in \operatorname{Bilin}\left(k_{1}, k_{2}\right)$ is called an $n^{\text {th }}$ order Hankel form, $n \geq 0$, if $\triangle^{I}(B)=$ 0 for every multi-index $I=\left(i_{1}, \ldots, i_{d}\right) \in Z_{+}^{d}$ with $|I|:=i_{1}+\cdots+i_{d}=n+1$.

Note that a form $B$ is a $0^{\text {th }}$ order Hankel form if $\triangle_{j}(B)=0, j=$ $1, \ldots, d$. This condition is equivalent to $B\left(p f_{1}, f_{2}\right)=B\left(f_{1}, p f_{2}\right)$ for all $p \in \mathrm{C}\left[z_{1}, \ldots, z_{d}\right]$ and $f_{i} \in H\left(k_{i}\right), i=1,2$. Thus, the $0^{\text {th }}$ order Hankel forms coincide with the usual Hankel forms. It also follows from the definition that a form $B$ is an $n^{\text {th }}$ order Hankel form, $n>0$, if for each $1 \leq j \leq d$, $\triangle_{j}(B)$ is a Hankel form of order $n-1$.

$\operatorname{Bilin}_{H S}\left(k_{1}, k_{2}\right)$ is invariant under the operators $R_{p}$ and $L_{p}, p \in \mathrm{C}\left[z_{1}, \ldots, z_{d}\right]$, and so the definition above yields subclasses of the Hilbert-Schmidt forms on $H\left(k_{1}\right) \times H\left(k_{2}\right)$. We will denote the $n^{\text {th }}$ order Hilbert-Schmidt Hankel forms $\mathscr{H}_{H S}^{n}\left(k_{1}, k_{2}\right), n \geq 0$. Note that $\mathscr{H}_{H S}^{n}\left(k_{1}, k_{2}\right) \subseteq \mathscr{H}_{H S}^{n+1}\left(k_{1}, k_{2}\right), n \geq 0$. Following the terminology in [9] we will call the forms in $\mathscr{H}_{H S}^{n}\left(k_{1}, k_{2}\right) \ominus \mathscr{H}_{H S}^{n-1}\left(k_{1}, k_{2}\right)$, $n>0$, the special $n^{\text {th }}$ order Hilbert-Schmidt Hankel forms.

\section{The symbols of the higher order Hilbert-Schmidt Hankel forms}

Define $V_{D}^{n}, n \geq 0$, to be the closure in $H\left(k_{1} \otimes k_{2}\right)$ of the space spanned by the range of the operator $\left(\mathbf{M}_{z}-\mathbf{M}_{w}\right)^{I}$ as $I$ ranges over all multi-indices in $Z_{+}^{d}$ with $|I|=n$. That is,

$$
\begin{aligned}
V_{D}^{n} & :=\bigvee_{|I|=n} \operatorname{Ran}\left(\mathbf{M}_{z}-\mathbf{M}_{w}\right)^{I} \\
& \left.=\sum_{|I|=n}(z-w)^{I} f_{I}(z, w): f_{I} \in H\left(k_{1} \otimes k_{2}\right)\right\}
\end{aligned}
$$

We will use the notation $\langle *\rangle$ to denote the ideal generated algebraically by the set of functions $\{*\}$. The spaces $V_{D}^{n}, n \geq 0$, form a decreasing chain of subspaces of $H\left(k_{1} \otimes k_{2}\right)$ and can be realized as the closure in $H\left(k_{1} \otimes k_{2}\right)$ of the ideal of polynomials generated by $(z-w)^{I}, I \in \mathrm{Z}_{+}^{d}$ and $|I|=n$. 
Proposition 2.1. Fix $n \geq 1$. Then

$$
V_{D}^{n}={\overline{\left\langle(z-w)^{I}: I \in Z_{+}^{d},|I|=n\right\rangle}}^{H\left(k_{1} \otimes k_{2}\right)} .
$$

Consequently, if $f \in V_{D}^{n}$ then $\left.\left(\frac{\partial}{\partial z}\right)^{I}\left(\frac{\partial}{\partial w}\right)^{J} f\right|_{z=w}=0$ for all multi-indices $I, J \in Z_{+}^{d}$ with $|I|+|J|<n$.

Proof. A function $f \in H\left(k_{1} \otimes k_{2}\right)$ is orthogonal to $\left\langle(z-w)^{I}: I \in\right.$ $\left.Z_{+}^{d},|I|=n\right\rangle$ if and only if $\left\langle\left(\mathbf{M}_{z}^{*}-\mathbf{M}_{w}^{*}\right)^{I} f, p\right\rangle=0$ for all polynomials $p$ and multi-indices $|I|=n$. By assumption, the polynomials are dense in $H\left(k_{1} \otimes k_{2}\right)$ and thus, $f$ is orthogonal to this ideal if and only if $f \in \operatorname{Ker}\left(\mathbf{M}_{z}-\mathbf{M}_{w}\right)^{I^{*}}$ for all multi-indices $|I|=n$. Therefore $V_{D}^{n \perp}=\left\langle(z-w)^{I}\left|I \in Z_{+}^{d},\right| I \mid=n\right\rangle^{\perp}$ and so the first statement now follows.

We note that a polynomial $p$ is in the ideal $\left\langle(z-w)^{I}: I \in Z_{+}^{d},|I|=n\right\rangle$ if and only if $\left.\left(\frac{\partial}{\partial z}\right)^{I}\left(\frac{\partial}{\partial w}\right)^{J} p\right|_{z=w}=0$ for all multi-indices $|I|+|J|<n$. This is easily verified by looking at the Taylor series. Now if $f \in V_{D}^{n}$ then, by definition, $f$ is the limit in $H\left(k_{1} \otimes k_{2}\right)$ of a sequence of polynomials $p_{m}$ satisfying $\left.\left(\frac{\partial}{\partial z}\right)^{I}\left(\frac{\partial}{\partial w}\right)^{J} p_{m}\right|_{z=w}=0$ for all multi-indices $|I|+|J|<n$. Using the fact that evaluation at $z \in \mathrm{D}^{2 d}$ is a bounded linear functional on $H\left(k_{1} \otimes k_{2}\right)$, one can show that the sequence $p_{m}$ converges uniformly to $f$ on compact subsets of $\mathrm{D}^{2 d}$ and thus, $\left.\left(\frac{\partial}{\partial z}\right)^{I}\left(\frac{\partial}{\partial w}\right)^{J} f\right|_{z=w}=0$ for all multi-indices $|I|+|J|<n$.

It follows from this proposition that $\cap_{n \geq 0} V_{D}^{n}=(0)$ and so we can decompose $H\left(k_{1} \otimes k_{2}\right)$ into the orthogonal sum

$$
H\left(k_{1} \otimes k_{2}\right)=V_{D}^{\perp} \oplus\left(V_{D} \ominus V_{D}^{2}\right) \oplus\left(V_{D}^{2} \ominus V_{D}^{3}\right) \oplus \cdots .
$$

Now we will identify the functions in $V_{D}^{n} \ominus V_{D}^{n+1}, n \geq 0$, with the symbols of the special $n^{\text {th }}$ order Hilbert-Schmidt Hankel forms. The following observation is simple but fundamental.

Proposition 2.2. If $B \in \operatorname{Bilin}_{H S}\left(k_{1}, k_{2}\right)$ and $I \in Z_{+}^{d}$ is a multi-index then $\pi\left(\triangle^{I}(B)\right)=\left(\mathbf{M}_{z}-\mathbf{M}_{w}\right)^{I^{*}} \pi(B)$. Consequently for $n \geq 0, B \in \mathscr{H}_{H S}^{n}\left(k_{1}, k_{2}\right)$ if and only if $\pi(B) \in V_{D}^{n+1^{\perp}}$. 
Proof. We observe that

$$
\begin{aligned}
\left(M_{z_{j}}-\right. & \left.M_{w_{j}}\right)^{*} \pi(B)(z, w) \\
& =\left\langle\pi(B),\left(M_{z_{j}}-M_{w_{j}}\right)\left(k_{1}(\cdot, z) \otimes k_{2}(\cdot, w)\right)\right\rangle \\
& =\left\langle\pi(B), M_{z_{j}} k_{1}(\cdot, z) \otimes k_{2}(\cdot, w)\right\rangle-\left\langle\pi(B), k_{1}(\cdot, z) \otimes M_{w_{j}} k_{2}(\cdot, w)\right\rangle \\
& =\overline{L_{z_{j}} B\left(k_{1}(\cdot, z), k_{2}(\cdot, w)\right)-R_{z_{j}} B\left(k_{1}(\cdot, z), k_{2}(\cdot, w)\right)} \\
& =\overline{\triangle_{j}(B)\left(k_{1}(\cdot, z), k_{2}(\cdot, w)\right)} \\
& =\pi\left(\triangle_{j}(B)\right)(z, w) .
\end{aligned}
$$

Since this holds for all $1 \leq j \leq d$ the first assertion follows.

By definition, a form $B \in \mathscr{H}_{H S}^{n}\left(k_{1}, k_{2}\right)$ if and only if $\triangle^{I}(B)=0$ for all $I \in \mathrm{Z}_{+}^{d}$ with $|I|=n+1$. The symbol map $\pi: \operatorname{Bilin}\left(k_{1}, k_{2}\right) \rightarrow H\left(k_{1} \otimes k_{2}\right)$ is 1-1 and so $B \in \mathscr{H}_{H S}^{n}\left(k_{1}, k_{2}\right)$ if and only if $\pi\left(\triangle^{I}(B)\right)=\left(\mathbf{M}_{z}-\mathbf{M}_{w}\right)^{I^{*}} \pi(B)=0$ for all $|I|=n+1$. Since $V_{D}^{n+1^{\perp}}=\bigcap_{|I|=n+1} \operatorname{Ker}\left(\mathbf{M}_{z}-\mathbf{M}_{w}\right)^{I^{*}}$ for all $n \geq 0$, the proof is complete.

It follows by Proposition 2.2 together with the fact that the symbol map $\pi: \operatorname{Bilin}\left(k_{1}, k_{2}\right) \rightarrow H\left(k_{1} \otimes k_{2}\right)$ is an isometry, that the functions in $V_{D}^{n} \ominus V_{D}^{n+1}$ are precisely the symbols of the forms in $\mathscr{H}_{H S}^{n}\left(k_{1}, k_{2}\right) \ominus \mathscr{H}_{H S}^{n-1}\left(k_{1}, k_{2}\right), n \geq 0$, where for $n=0$ we define $\mathscr{H}_{H S}^{-1}\left(k_{1}, k_{2}\right)=(0)$. Since $V_{D}^{n+1} \perp=\left(V_{D}^{1}\right)^{\perp} \oplus$ $\left(V_{D}^{1} \ominus V_{D}^{2}\right) \oplus \cdots \oplus\left(V_{D}^{n} \ominus V_{D}^{n+1}\right)$, the corollary below is immediate.

Corollary 2.3. Let $B \in \operatorname{Bilin}_{H S}\left(k_{1}, k_{2}\right)$ and $n \geq 0$.

(i) $B \in \mathscr{H}_{H S}^{n}\left(k_{1}, k_{2}\right) \ominus \mathscr{H}_{H S}^{n-1}\left(k_{1}, k_{2}\right)$ if and only if $\pi(B) \in V_{D}^{n} \ominus V_{D}^{n+1}$.

(ii) $B \in \mathscr{H}_{H S}^{n}\left(k_{1}, k_{2}\right)$ if and only if $\pi(B) \in\left(V_{D}^{1}\right)^{\perp} \oplus\left(V_{D}^{1} \ominus V_{D}^{2}\right) \oplus \cdots \oplus$ $\left(V_{D}^{n} \ominus V_{D}^{n+1}\right)$.

\section{An $n^{\text {th }}$ order restriction result for the polydisk}

For $\alpha=\left(\alpha_{1}, \ldots, \alpha_{d}\right)$, with $\alpha_{j}>0, j=1, \ldots, d$, we define the kernel $k_{\alpha}$ on $\mathrm{D}^{d} \times \mathrm{D}^{d}$ by

$$
k_{\alpha}(z, \lambda)=\frac{1}{(1-z \bar{\lambda})^{\alpha}}:=\frac{1}{\left(1-z_{1} \overline{\lambda_{1}}\right)^{\alpha_{1}}} \ldots \frac{1}{\left(1-z_{d} \overline{\lambda_{d}}\right)^{\alpha_{d}}} .
$$

If $\alpha=I_{0}:=(1, \ldots, 1)$ then $H\left(k_{\alpha}\right)$ is isometrically equal to $H^{2}\left(\mathrm{D}^{d}\right)$, the Hardy space of the polydisk. If $\alpha_{j}>1, j=1, \ldots, d$, then $H\left(k_{\alpha}\right)$ is isometrically equal to the d-fold Hilbert space tensor product of the standard weighted Bergman spaces $A^{2, \alpha_{j}}, j=1, \ldots, d$. Hence, $H\left(k_{\alpha}\right)$ can be realized as holomorphic functions on the polydisk which are square integrable with respect to the $d$-fold tensor of weighted area measures. 
To be specific and fix notation, for $\gamma=\left(\gamma_{1}, \ldots, \gamma_{d}\right)$ with $\gamma_{j}>-1, j=$ $1, \ldots, d$, we define the measure $\mu_{\gamma}$ by

$$
\begin{aligned}
& d \mu_{\gamma}(z) \\
& =\pi^{-d}\left(\gamma_{1}+1\right) \ldots\left(\gamma_{d}+1\right)\left(1-\left|z_{1}\right|^{2}\right)^{\gamma_{1}} \ldots\left(1-\left|z_{d}\right|^{2}\right)^{\gamma_{d}} d A\left(z_{1}\right) \ldots d A\left(z_{d}\right) .
\end{aligned}
$$

Here $d A$ denotes the element of area measure on the plane. The space $A^{2, \gamma}$ is defined to be $L^{2}\left(\mathrm{D}^{d}, \mu_{\gamma}\right) \cap \operatorname{Hol}\left(\mathrm{D}^{d}\right)$. The reproducing kernel for $A^{2, \gamma}$ is $k_{\gamma+2 I_{0}}$. Thus, if $\alpha_{j}>1, j=1, \ldots, d$, then $H\left(k_{\alpha}\right)=A^{2, \alpha-2 I_{0}}$ and the norms are the same.

If $0<\alpha_{j}<1$ for some $1 \leq j \leq d$, then the space $H\left(k_{\alpha}\right)$ is called a Dirichlet-type space and coincides with the space of functions $f$, holomorphic on $\mathrm{D}^{d}$, such that $D^{I_{0}} f \in A^{2, \alpha}$. Dirichlet spaces in one variable and Hankel operators defined on them have been studied extensively by $\mathrm{Wu}$ and the second author; more references can be found in [17], [22].

Now let $k_{\beta}$ be another kernel of the form in (3.1) with $\beta=\left(\beta_{1}, \ldots, \beta_{d}\right)$, $\beta_{j}>0$. We will describe the functions in $V_{D}^{n} \ominus V_{D}^{n+1} \subseteq H\left(k_{\alpha} \otimes k_{\beta}\right)$ by

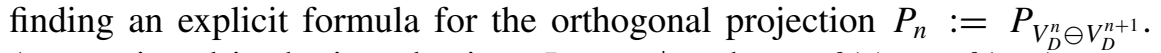
As mentioned in the introduction, $P_{0}=r^{*} r$ where $r f(z)=f(z, z)$ maps $H\left(k_{\alpha} \otimes k_{\beta}\right)$ onto $H\left(k_{\alpha+\beta}\right)$, [20], [2], [6], [8], [21]. In the case where $\alpha_{j}+\beta_{j}>1$, $j=1, \ldots, d$, this yields

$$
\left(P_{0} f\right)(z, w)=\int_{D^{d}} \frac{f(\lambda, \lambda)}{(1-z \bar{\lambda})^{\alpha}(1-w \bar{\lambda})^{\beta}} d \mu_{\alpha+\beta-2 I_{0}}(\lambda) .
$$

In one variable, $P_{1}$ was determined in [14] for the kernels $k_{\alpha}$ and $k_{\beta}$ with $\alpha, \beta \geq 1$. The formula is

$$
\left(P_{1} f\right)(z, w)=v_{1}^{2}(z-w) \int_{\mathrm{D}} \frac{\left(\partial_{1} f-\partial_{2} f\right)(\lambda, \lambda)}{(1-z \bar{\lambda})^{\alpha+1}(1-w \bar{\lambda})^{\beta+1}} d \mu_{\alpha+\beta}(\lambda)
$$

where $v_{1}=\|(z-w)\|_{H\left(k_{\alpha} \otimes k_{\beta}\right)}$. We now extend this result to the higher order case and to the polydisk.

We now introduce the appropriate generalization of the transvectants introduced by Janson and Peetre in [9]. For a multi-index $I \in \mathrm{Z}_{+}^{d}$ define the partial differential operators $\mathscr{F}_{I}$ by

$$
\mathscr{J}_{I}=\sum_{K+J=I} \frac{(-1)^{|J|} I !}{K ! J !(\alpha)_{K}(\beta)_{J}}\left(\frac{\partial}{\partial z}\right)^{K}\left(\frac{\partial}{\partial w}\right)^{J}
$$

where for $\gamma=\left(\gamma_{1}, \ldots, \gamma_{d}\right)$ and $J=\left(j_{1}, \ldots, j_{d}\right) \in Z_{+}^{d}$,

$$
(\gamma)_{J}:=\left(\gamma_{1}\right)_{j_{1}} \ldots\left(\gamma_{1}\right)_{j_{d}}
$$


and

$$
\left(\gamma_{i}\right)_{j_{i}}:=\frac{\Gamma\left(\gamma_{i}+j_{i}\right)}{\Gamma\left(\gamma_{i}\right)}=\gamma_{i}\left(\gamma_{i}+1\right) \ldots\left(\gamma_{i}+j_{i}-1\right), \quad i=1, \ldots, d .
$$

Define the restriction operator

$$
r_{I}=r \mathscr{J}_{I}: H\left(k_{\alpha} \otimes k_{\beta}\right) \rightarrow \operatorname{Hol}\left(\mathrm{D}^{d}\right) .
$$

We record the following observation about the map $r_{I}$ for future use.

Proposition 3.1. If $g(z, w)=(z-w)^{I} f(z, w)$ where $f \in H\left(k_{\alpha} \otimes k_{\beta}\right)$, then $r_{I}(g)=\left\|(z-w)^{I}\right\|_{H\left(k_{\alpha} \otimes k_{\beta}\right)}^{2} r(f)$. Also, $r_{J}(g)=0$ for all multi-indices $|J|=|I|$ with $J \neq I$.

Proof. Let $K, L \in Z_{+}^{d}$ with $|K|+|L|=|I|$. If $f \in \operatorname{Hol}\left(\mathrm{D}^{2 d}\right)$ then we have that

$$
r\left(\left(\frac{\partial}{\partial z}\right)^{K}\left(\frac{\partial}{\partial w}\right)^{L}(z-w)^{I} f(z, w)\right)= \begin{cases}(-1)^{|L|} I ! r(f) & K+L=I \\ 0 & K+L \neq I\end{cases}
$$

Thus,

$$
\begin{aligned}
r_{I}(g) & =\sum_{K+L=I} \frac{(-1)^{L} I !}{K ! L !(\alpha)_{K}(\beta)_{L}} r\left(\frac{\partial}{\partial z}\right)^{K}\left(\frac{\partial}{\partial w}\right)^{J}(g) \\
& =\sum_{K+L=I} \frac{(I !)^{2}}{K ! L !(\alpha)_{K}(\beta)_{L}} r(f) \\
& =\sum_{K+L=I} \frac{(I !)^{2}}{(K !)^{2}(L !)^{2}} \frac{K !}{(\alpha)_{K}} \frac{L !}{(\beta)_{L}} r(f) \\
& =\sum_{K+L=I} \frac{(I !)^{2}}{(K !)^{2}(L !)^{2}}\left\|z^{K}\right\|_{H\left(k_{\alpha}\right)}^{2}\left\|w^{L}\right\|_{H\left(k_{\beta}\right)}^{2} r(f) \\
& =\sum_{K+L=I} \frac{(I !)^{2}}{(K !)^{2}(L !)^{2}}\left\|z^{K} w^{L}\right\|_{H\left(k_{\alpha} \otimes k_{\beta}\right)}^{2} r(f) \\
& =\left\|(z-w)^{I}\right\|_{H\left(k_{\alpha} \otimes k_{\beta}\right)}^{2} r(f)
\end{aligned}
$$

Similarly, if $|J|=|I|$ but $J \neq I$ then every term of the form $r\left(\left(\frac{\partial}{\partial z}\right)^{K}\left(\frac{\partial}{\partial w}\right)^{L} g\right)$ with $K+L=J$ is zero and thus, $r_{J}(g)=0$.

To find the null space and range of the map $r_{I}$ we first need to determine the action of $r_{I}$ on kernel functions. A direct computation shows that for all 
$z, w, \lambda \in \mathrm{D}^{d}$

$$
r_{I}\left(k_{\alpha}(\cdot, z) \otimes k_{\beta}(\cdot, w)\right)(\lambda)=\frac{(\bar{z}-\bar{w})^{I}}{(1-\bar{z} \lambda)^{\alpha+I}(1-\bar{w} \lambda)^{\beta+I}} .
$$

Consequently, if $\mathscr{J}_{I}^{*}$ denotes the formal (Hilbert space) adjoint of $\mathscr{J}_{I}$, i.e., for $f \in H\left(k_{\alpha} \otimes k_{\beta}\right)$,

$$
\left(\mathscr{J}_{I}^{*} f\right)(z, w)=\left\langle f, \mathscr{J}_{I}\left(k_{\alpha}(\cdot, z) \otimes k_{\beta}(\cdot, w)\right)\right\rangle
$$

then for $\lambda \in \mathrm{D}^{d}$,

$$
\begin{aligned}
\mathscr{J}_{I}^{*}\left(k_{\alpha}(\cdot, \lambda) \otimes k_{\beta}(\cdot, \lambda)\right)(z, w) & =\left\langle k_{\alpha}(\cdot, \lambda) \otimes k_{\beta}(\cdot, \lambda), \mathscr{J}_{I}\left(k_{\alpha}(\cdot, z) \otimes k_{\beta}(\cdot, w)\right)\right\rangle \\
& =\overline{r_{I}\left(k_{\alpha}(\cdot, z) \otimes k_{\beta}(\cdot, w)\right)(\lambda)} \\
& =(z-w)^{I} k_{\alpha+I}(z, \lambda) k_{\beta+I}(w, \lambda) .
\end{aligned}
$$

Now let $f \in H\left(k_{\alpha} \otimes k_{\beta}\right)$ and $\lambda \in \mathrm{D}^{d}$. Then

$$
\begin{aligned}
\left(r_{I} f\right)(\lambda)=\left(\mathscr{J}_{I} f\right)(\lambda, \lambda) & =\left\langle\mathscr{J}_{I} f, k_{\alpha}(\cdot, \lambda) \otimes k_{\beta}(\cdot, \lambda)\right\rangle \\
& =\left\langle f,(z-w)^{I} k_{\alpha+I}(\cdot, \lambda) \otimes k_{\beta+I}(\cdot, \lambda)\right\rangle .
\end{aligned}
$$

It follows that

$$
\left(\operatorname{Ker} r_{I}\right)^{\perp}=\bigvee\left\{\frac{(z-w)^{I}}{(1-z \bar{\lambda})^{\alpha+I}(1-w \bar{\lambda})^{\beta+I}}: \lambda \in \mathrm{D}^{d}\right\} .
$$

We now show that for all $|I| \geq 0$ the range of the map $r_{I}$ is $H\left(k_{\alpha+\beta+2 I}\right)$. By Proposition 3.1,

$$
\begin{aligned}
r_{I} \mathscr{J}_{I}^{*}\left(k_{\alpha}(\cdot, \lambda) \otimes k_{\beta}(\cdot, \lambda)\right) & =r_{I}\left((z-w)^{I} k_{\alpha+I}(\cdot, \lambda) \otimes k_{\beta+I}(\cdot, \lambda)\right) \\
& =\left\|(z-w)^{I}\right\|_{H\left(k_{\alpha} \otimes k_{\beta}\right)}^{2} k_{\alpha+\beta+2 I}(\cdot, \lambda) .
\end{aligned}
$$

It follows that $r_{I}$ maps a dense subset of $\oplus_{|I|=n}\left(\operatorname{Ker} r_{I}\right)^{\perp}$ onto a dense subset of $H\left(k_{\alpha+\beta+2 I}\right)$. We now make a straightforward calculation to show that after normalizing, $r_{I}$ becomes a partial isometry of $H\left(k_{\alpha} \otimes k_{\beta}\right)$ mapping (necessarily) onto $H\left(k_{\alpha+\beta+2 I}\right)$.

THEOREM 3.2. Let $I \in \mathrm{Z}_{+}^{d}$. The map $r_{I}$ maps $H\left(k_{\alpha} \otimes k_{\beta}\right)$ onto $H\left(k_{\alpha+\beta+2 I}\right)$ and satisfies $r_{I} r_{I}^{*}=v_{I}^{2} 1$ where $v_{I}=\left\|(z-w)^{I}\right\|_{H\left(k_{\alpha} \otimes k_{\beta}\right)}$. Consequently, $P_{I}=$ $v_{I}^{-2} r_{I}^{*} r_{I}$ is the orthogonal projection of $H\left(k_{\alpha} \otimes k_{\beta}\right)$ onto $\left(\operatorname{Ker} r_{I}\right)^{\perp}$. 
Proof. Fix a finite subset $\left\{\lambda_{1}, \ldots, \lambda_{m}\right\} \subseteq \mathrm{D}^{d}$ and scalars $c_{1}, \ldots, c_{m}$. Then

$$
\begin{aligned}
& \left\|r_{I}\left(\sum_{j}^{m} c_{j} \mathscr{F}_{I}^{*}\left(k_{\alpha}\left(\cdot, \lambda_{j}\right) \otimes k_{\beta}\left(\cdot, \lambda_{j}\right)\right)\right)\right\|_{H\left(k_{\alpha+\beta+2 l}\right)}^{2} \\
& \quad=v_{I}^{4}\left\|\sum_{j}^{m} c_{j} k_{\alpha+\beta+2 I}\left(\cdot, \lambda_{j}\right)\right\|_{H\left(k_{\alpha+\beta+2 l}\right)}^{2}=v_{I}^{4} \sum_{i, j} \overline{c_{i}} c_{j} k_{\alpha+\beta+2 I}\left(\lambda_{i}, \lambda_{j}\right) .
\end{aligned}
$$

On the other hand,

$$
\begin{aligned}
\left\|\sum_{j}^{m} c_{j} \mathscr{J}_{I}^{*}\left(k_{\alpha}\left(\cdot, \lambda_{j}\right) \otimes k_{\beta}\left(\cdot, \lambda_{j}\right)\right)\right\|_{H\left(k_{\alpha} \otimes k_{\beta}\right)}^{2} \\
\quad=\sum_{i, j} c_{j} \overline{c_{j}}\left\langle\mathscr{J}_{I} \mathscr{J}_{I}^{*}\left(k_{\alpha}\left(\cdot, \lambda_{j}\right) \otimes k_{\beta}\left(\cdot, \lambda_{j}\right)\right), k_{\alpha}\left(\cdot, \lambda_{i}\right) \otimes k_{\beta}\left(\cdot, \lambda_{i}\right)\right\rangle \\
=v_{I}^{4} \sum_{i, j} \overline{c_{i}} c_{j} k_{\alpha+\beta+2 I}\left(\lambda_{i}, \lambda_{j}\right) .
\end{aligned}
$$

Thus,

$$
\begin{aligned}
& \left\|r_{I}\left(\sum_{j}^{m} c_{j} \mathscr{J}_{I}^{*}\left(k_{\alpha}\left(\cdot, \lambda_{j}\right) \otimes k_{\beta}\left(\cdot, \lambda_{j}\right)\right)\right)\right\|_{H\left(k_{\alpha+\beta+2 I}\right)} \\
& \quad=v_{I}\left\|\sum_{j}^{m} c_{j} \mathscr{F}_{I}^{*}\left(k_{\alpha}\left(\cdot, \lambda_{j}\right) \otimes k_{\beta}\left(\cdot, \lambda_{j}\right)\right)\right\|_{H\left(k_{\alpha} \otimes k_{\beta}\right)} .
\end{aligned}
$$

This holds for all finite subsets $\left(\lambda_{i}\right) \subseteq \mathrm{D}^{d}$ and scalars $c_{i} \in \mathrm{C}$. Since finite linear combinations of the functions $\mathscr{J}_{I}^{*}\left(k_{\alpha}(\cdot, \lambda) \otimes k_{\beta}(\cdot, \lambda)\right)$ are dense in $\left(\operatorname{Ker} r_{I}\right)^{\perp}$ it follows that for all $f \in\left(\operatorname{Ker} r_{I}\right)^{\perp}$,

$$
\left\|r_{I} f\right\|_{H\left(k_{\alpha+\beta+2 I}\right)}=v_{I}\|f\|_{H\left(k_{\alpha} \otimes k_{\beta}\right)}
$$

Thus $v_{I}^{-1} r_{I}$ is a partially isometric mapping of $H\left(k_{\alpha} \otimes k_{\beta}\right)$ onto $H\left(k_{\alpha+\beta+2 I}\right)$. Therefore, $P_{I}=v_{I}^{-2} r_{I}^{*} r_{I}$ is the orthogonal projection of $H\left(k_{\alpha} \otimes k_{\beta}\right)$ onto $\left(\operatorname{Ker} r_{I}\right)^{\perp}$.

We now use the maps $r_{I},|I|=n$, to give a formula for the orthogonal projection onto $V_{D}^{n} \ominus V_{D}^{n+1}$. First observe that if $|I|=|J|$ with $I \neq J$ then $\left(\operatorname{Ker} r_{I}\right)^{\perp} \perp\left(\operatorname{Ker} r_{J}\right)^{\perp}$. To see this we note, by Proposition 3.1, that $r_{J}(z-w)^{I} f=0$ for every $f \in H\left(k_{\alpha} \otimes k_{\beta}\right)$. Thus for all $\lambda \in \mathrm{D}^{d}$.

$$
r_{J}\left((z-w)^{I} k_{\alpha+I}(\cdot, \lambda) \otimes k_{\beta+I}(\cdot, \lambda)\right)=0 .
$$


By (3.4), $\left(\operatorname{Ker} r_{I}\right)^{\perp}$ is spanned by the functions $(z-w)^{I} k_{\alpha+I}(\cdot, \lambda) \otimes k_{\beta+I}(\cdot, \lambda)$, $\lambda \in \mathrm{D}^{d}$. Thus, for all $I, J,|I|=|J|, I \neq J$ we have $r_{J}\left(\operatorname{Ker} r_{I}\right)^{\perp}=(0)$.

Theorem 3.3. For $n \geq 0, V_{D}^{n} \ominus V_{D}^{n+1}=\bigoplus_{|I|=n}\left(\operatorname{Ker} r_{I}\right)^{\perp}$.

Proof. Fix $n \geq 0$ and set

$$
\mathscr{L}_{n}=\bigoplus_{|I|=n}\left(\operatorname{Ker} r_{I}\right)^{\perp} .
$$

Then by (3.4),

$$
\mathscr{L}_{n}=\bigvee_{|I|=n}\left\{\frac{(z-w)^{I}}{(1-z \bar{\lambda})^{\alpha+I}(1-w \bar{\lambda})^{\beta+I}}: \lambda \in \mathrm{D}^{d}\right\} .
$$

It is clear that $\mathscr{L}_{n} \subseteq V_{D}^{n}$. If $f \in V_{D}^{n+1}$ then by Proposition $2.1, r_{I}(f)=0$ for every multi-index $I$ with $|I|=n$. Thus $V_{D}^{n+1} \subseteq \operatorname{Ker} r_{I}$ for all $|I|=n$. Hence $\mathscr{L}_{n} \subseteq V_{D}^{n+1^{\perp}}$ and so $\mathscr{L}_{n} \subseteq V_{D}^{n} \ominus V_{D}^{n+1}$.

Let $g \in V_{D}^{n} \ominus V_{D}^{n+1}$ and suppose that $g \perp \mathscr{L}_{n}$. We can write $g(z, w)=$ $\sum_{|J|=n}(z-w)^{J} f_{J}(z, w)$ where for each multi-index $|J|=n, f_{J}$ is holomorphic on $\mathrm{D}^{2 d}$. Since $g \perp \mathscr{L}_{n}, r_{I}(g)=0$ for all $|I|=n$. Now fix a multiindex $I$ with $|I|=n$. By Proposition 3.1, $0=r_{I}(g)=r_{I}\left((z-w)^{I} f_{I}(z, w)\right)=$ $\left\|(z-w)^{I}\right\|_{H\left(k_{\alpha} \otimes k_{\beta}\right)} r\left(f_{I}\right)$ and so we may write $f_{I}=\sum_{j=1}^{d}\left(z_{j}-w_{j}\right) f_{I, j}$ where $f_{I, j} \in \operatorname{Hol}\left(\mathrm{D}^{2 d}\right)$. It follows that $g(z, w)=\sum_{|J|=n+1}(z-w)^{J} g_{J}(z, w)$ where $g_{J} \in \operatorname{Hol}\left(\mathrm{D}^{2 d}\right)$ and a standard limiting argument shows that $g \in V_{D}^{n+1}$. But since $g \in\left(V_{D}^{n+1}\right)^{\perp}, g=0$. Therefore, $\mathscr{L}_{n}=V_{D}^{n} \ominus V_{D}^{n+1}$.

We now see that we have isolated the same subspaces as those which can be identified by analyzing the group action.

COROLlaRy 3.4. The irreducible components of the natural action of the Möbius group on $H\left(k_{\alpha} \otimes k_{\beta}\right)=H\left(k_{\alpha}\right) \otimes H\left(k_{\beta}\right)$ are the spaces of symbols of special Hankel forms of order $n$.

Proof. First suppose $d=1$. In the proof of the theorem we identified the space of such symbols with the closed span of $\mathscr{L}_{n}$. Now note that if, instead of using $\mathscr{L}_{n}$, in which the parameter $\lambda$ varies over $\mathrm{D}$ (we are in the case $d=1$ ), we work with $\mathcal{M}_{n}$ which we define by now allowing $\lambda$ to only vary over $\partial \mathrm{D}$, we obtain the same closed span. This holds because taking weighted averages (i.e. Poisson integrals) of elements of $\mathscr{M}_{n}$ generates $\mathscr{L}_{n}$. In Section 1 [24] Zhang shows that the irreducible components are the spans of sets $\mathscr{M}_{n}$.

For general $d$, recall that every biholomorphic automorphism of $\mathrm{D}^{d}=$ $\mathrm{D} \times \cdots \times \mathrm{D}$ is induced by Möbius maps of the individual D's [20]. Hence 
the $d$ dimensional version of the Corollary follows from the 1 dimensional version.

It follows immediately from Theorems 3.2 and 3.3 that, denoting the orthogonal projection of $H\left(k_{\alpha} \otimes k_{\beta}\right)$ onto $V_{D}^{n} \ominus V_{D}^{n+1}$ by $P_{n}$, we have, for $n \geq 0$,

$$
P_{n}=\sum_{|I|=n} v_{I}^{-2} r_{I}^{*} r_{I}
$$

where $v_{I}=\left\|(z-w)^{I}\right\|_{H\left(k_{\alpha} \otimes k_{\beta}\right)}, I \in \mathbf{Z}_{+}^{d}$.

We can now build the map which intertwines the compression of multiplication operators to $V_{D}^{n} \ominus V_{D}^{n+1}$ with a direct sum of single variable multiplication operators

Corollary 3.5. Fix $n \geq 0$. The map $R_{n} f:=\oplus \sum_{|I|=n} v_{I}^{-1} r_{I}(f)$ establishes an isometric isomorphism between the spaces $V_{D}^{n} \ominus V_{D}^{n+1} \subseteq H\left(k_{\alpha} \otimes k_{\beta}\right)$ and $\oplus_{|I|=n} H\left(k_{\alpha+\beta+2 I}\right)$. Furthermore, for all $p \in \mathrm{C}\left[z_{1}, \ldots, z_{d}, w_{1}, \ldots, w_{d}\right]$

$$
R_{n}\left(P_{n} M_{p} \mid V_{D}^{n} \ominus V_{D}^{n+1}\right)=\left(\bigoplus_{|I|=n} M_{r(p)}\right) R_{n}
$$

Proof. Fix $I \in Z_{+}^{d}$. By Theorem 3.2, the map $v_{I}^{-1} r_{I}^{*}: H\left(k_{\alpha+\beta+2 I}\right) \rightarrow$ $H\left(k_{\alpha} \otimes k_{\beta}\right)$ is an isometry with range equal to $\left(\operatorname{Ker} r_{I}\right)^{\perp}$. By Theorem 3.3, $V_{D}^{n} \ominus V_{D}^{n+1}=\bigoplus_{|I|=n}\left(\operatorname{Ker} r_{I}\right)^{\perp}$ and thus, the map $R_{n}: H\left(k_{\alpha} \otimes k_{\beta}\right) \rightarrow$ $\bigoplus_{|I|=n} H\left(k_{\alpha+\beta+2 I}\right)$ defined above satisfies $R_{n}^{*} R_{n}=P_{n}$. It follows that $R_{n}$ is co-isometric with kernel $\left(V_{D}^{n} \ominus V_{D}^{n+1}\right)^{\perp}$. The first statement now follows. To see the intertwining property of $R_{n}$ we use Proposition 3.1. If $f(z, w)=$ $\sum_{|I|=n}(z-w)^{I} f_{I}(z, w) \in V_{D}^{n}$ then

$$
\begin{aligned}
R_{n} M_{z_{i}} f & =\left(\oplus \sum_{|I|=n} v_{I}^{-1} r_{I}\right)\left(M_{z_{i}} f\right)=\oplus \sum_{|I|=n} v_{I}^{-1} r_{I}(z-w)^{I} z_{i} f_{I} \\
& =\oplus \sum_{|I|=n} v_{I} z_{i} r f_{I}=\oplus \sum_{|I|=n} z_{i} v_{I}^{-1} r_{I}(z-w)^{I} f_{I} \\
& =\left(\oplus \sum_{|I|=n} z_{i} v_{I}^{-1} r_{I}\right) f=\left(\bigoplus_{|I|=n} z_{i} \otimes I\right) R_{n} f .
\end{aligned}
$$

Since $P_{n} M_{z_{i}} P_{n}=P_{n} M_{w_{i}} P_{n}, i=1, \ldots, d$, the intertwining property of the map $R_{n}$ is now established.

Both $V_{D}^{n} \ominus V_{D}^{n+1}$ and $\bigoplus_{|I|=n} H\left(k_{\alpha+\beta+2 I}\right)$ are Hilbert modules over the polydisk algebra $\mathrm{A}\left(\mathrm{D}^{2 d}\right)$. In the first case the module action is $(a, v) \rightarrow P_{n}(a v)$, 
in the second case the action is

$$
\left(a,\left(h_{1}, \ldots, h_{m(I)}\right)\right) \rightarrow\left(r(a) h_{1}, \ldots, r(a) h_{m(I)}\right) .
$$

The corollary establishes that we have two equivalent Hilbert modules:

$$
V_{D}^{n} \ominus V_{D}^{n+1} \cong \bigoplus_{|I|=n} H\left(k_{\alpha+\beta+2 I}\right)
$$

We should also note, however, something we have not established. Fix $n \geq 1$ and set

$$
W^{n}=V_{D}^{\perp} \oplus\left(V_{D} \ominus V_{D}^{2}\right) \oplus\left(V_{D}^{2} \ominus V_{D}^{3}\right) \oplus \cdots \oplus\left(V_{D}^{n} \ominus V_{D}^{n+1}\right) .
$$

Thus $Q_{n}:=P_{0}+\cdots+P_{n}$ is the orthogonal projection onto $W^{n} . W^{n}$ is a Hilbert module over $\mathrm{A}\left(\mathrm{D}^{2 d}\right)$ with the action $(a, v) \rightarrow Q_{n}(a v)$. However, although (3.6) gives a realization of $W^{n}$ as an orthogonal direct sum of spaces and each of these spaces carries a Hilbert module structure, (3.6) is not a module direct sum. That is, the module action on $W^{n}$ is not the direct sum of the module actions on the $V_{D}^{j} \ominus V_{D}^{j+1}$. In formulas, if $v \in W^{n}$ splits as $v=v_{0}+\cdots+v_{n}$ and $a \in \mathrm{A}\left(\mathrm{D}^{2 d}\right)$ then $Q_{n}(a v)=\sum_{j} P_{j}\left(a \sum_{k} v_{k}\right)=\sum_{j, k} P_{j}\left(a v_{k}\right)$, however the off diagonal terms need not vanish and the previous sum need not equal $\sum_{r} P_{r}\left(a v_{r}\right)$. In particular, the structure we have developed here does not let us fully describe the module structure of $W^{n}$. Modules such as $W^{n}$ are considered systematically in [5].

We now turn our attention to higher order Hilbert-Schmidt Hankel forms on $H\left(k_{\alpha}\right) \times H\left(k_{\beta}\right)$ and deduce the representation given in [9] for $d=1$. If $b \in H\left(k_{\alpha+\beta+2 I}\right)$ then,

$$
\begin{aligned}
\left(r_{I}^{*} b\right)(z, w) & =\left\langle b, r_{I}\left(k_{\alpha}(\cdot, z) \otimes k_{\beta}(\cdot, w)\right\rangle_{H\left(k_{\alpha+\beta+2 I}\right)}\right. \\
& =(z-w)^{I}\left\langle b, k_{\alpha+I}(\cdot, z) k_{\beta+I}(\cdot, w)\right\rangle_{H\left(k_{\alpha+\beta+2 I}\right)} .
\end{aligned}
$$

Therefore, every function $a \in V_{D}^{n} \ominus V_{D}^{n+1}$ is completely determined by a vector-valued function $\mathbf{b}=\left(b_{I}\right)_{|I|=n} \in \bigoplus_{|I|=n} H\left(k_{\alpha+\beta+2 I}\right)$ via the formula

$$
\begin{aligned}
a(z, w) & =\left(R_{n}^{*} \mathbf{b}\right)(z, w)=\left(\sum_{|I|=n} v_{I}^{-1} r_{I}^{*} b_{I}\right)(z, w) \\
& =\sum_{|I|=n} v_{I}^{-1}(z-w)^{I}\left\langle b_{I}, k_{\alpha+I}(\cdot, z) k_{\beta+I}(\cdot, w)\right\rangle_{H\left(k_{\alpha+\beta+2 l}\right)}
\end{aligned}
$$

and we have $\|a\|^{2}=\sum_{|I|=n}\left\|b_{I}\right\|_{H\left(k_{\alpha+\beta+2 I}\right)}^{2}$. Let $a \in V_{D}^{n} \ominus V_{D}^{n+1}$ be given by the formula above and let $B$ be the Hilbert-Schmidt form on $H\left(k_{\alpha}\right) \times H\left(k_{\beta}\right)$ 
with symbol $a$. Then,

$$
B(f, g)=\langle f \otimes g, a\rangle=\sum_{|I|=n} v_{I}^{-1}\left\langle r_{I}(f \otimes g), b_{I}\right\rangle_{H\left(k_{\alpha+\beta+I}\right)} .
$$

By Corollary 2.3, $B$ is a special Hilbert-Schmidt Hankel form of order $n$, i.e., $B \in \mathscr{H}_{H S}^{n} \ominus \mathscr{H}_{H S}^{n-1}$, and furthermore, every form in $\mathscr{H}_{H S}^{n} \ominus \mathscr{H}_{H S}^{n-1}$ has this form. We record this below.

Theorem 3.6. Fix $n \geq 0$ and let $\mathbf{b}=\left(b_{I}\right)_{|I|=n} \in \bigoplus_{|I|=n} H\left(k_{\alpha+\beta+2 I}\right)$. Let $H_{\mathbf{b}}^{n}$ be the form on $H\left(k_{\alpha}\right) \times H\left(k_{\beta}\right)$ defined by

$$
H_{\mathbf{b}}^{n}(f, g)=\sum_{|I|=n}\left\langle r_{I}(f \otimes g), b_{I}\right\rangle_{H\left(k_{\alpha+\beta+2 I}\right)} .
$$

Then $H_{\mathbf{b}}^{n}$ is in $\mathscr{H}_{H S}^{n}\left(k_{\alpha}, k_{\beta}\right) \ominus \mathscr{H}_{H S}^{n-1}\left(k_{\alpha}, k_{\beta}\right)$ and

$$
\left\|H_{\mathbf{b}}^{n}\right\|_{H S}^{2}=\sum_{|I|=n} v_{I}^{2}\left\|b_{I}\right\|_{H\left(k_{\alpha+\beta+2 I}\right)}^{2}
$$

Furthermore,

$$
\mathscr{H}_{H S}^{n}\left(k_{\alpha}, k_{\beta}\right) \ominus \mathscr{H}_{H S}^{n-1}\left(k_{\alpha}, k_{\beta}\right)=\left\{H_{\mathbf{b}}^{n} \mid \mathbf{b} \in \bigoplus_{|I|=n} H\left(k_{\alpha+\beta+2 I}\right)\right\} .
$$

\section{An $n^{\text {th }}$ order restriction result for the ball}

Let $\mathrm{B}_{d}$ denote the unit ball in $\mathrm{C}^{d}$. The kernels we will be working with are defined on $\mathrm{B}_{d} \times \mathrm{B}_{d}$, for $\alpha>0$, by

$$
k_{\alpha}(z, \lambda)=\left(\frac{1}{1-\langle z, \lambda\rangle_{d}}\right)^{\alpha}
$$

where $\langle\cdot, \cdot\rangle_{d}$ denotes the inner product in $\mathrm{C}^{d}$. Given two parameters, $\alpha, \beta>0$, the space $H\left(k_{\alpha} \otimes k_{\beta}\right)$ is defined to be the Hilbert space of holomorphic functions on $\mathrm{B}_{d} \times \mathrm{B}_{d}$ with orthonormal basis

$$
\left\{\sqrt{\frac{I ! J !}{(\alpha)_{|I|}(\beta)_{|J|}}} z^{I} w^{J}: I, J \in Z_{+}^{d}\right\} .
$$

Alternatively, $H\left(k_{\alpha} \otimes k_{\beta}\right)$ is the Hilbert space tensor product of the spaces $H\left(k_{\alpha}\right)$ and $H\left(k_{\beta}\right)$ identified concretely as a space of holomorphic functions 
on $\mathrm{B}_{d} \times \mathrm{B}_{d}$. As shown in [21] $H\left(k_{\alpha} \otimes k_{\beta}\right)$ is a reproducing kernel Hilbert space with reproducing kernel

$$
\left(k_{\alpha} \otimes k_{\beta}\right)(z, w, \lambda, \zeta)=\left(\frac{1}{1-\langle z, \lambda\rangle_{d}}\right)^{\alpha}\left(\frac{1}{1-\langle w, \zeta\rangle_{d}}\right)^{\beta}
$$

for $z, w, \lambda, \zeta \in \mathbf{B}_{d}$.

The subspace $V_{D}^{n}, n \geq 0$, is defined to be the closure in $H\left(k_{\alpha} \otimes k_{\beta}\right)$ of the ideal of polynomials generated by $\left\{(z-w)^{N}:|N|=n\right\}$. The family $\left\{V_{D}^{n}\right\}_{n \geq 0}$ forms a decreasing chain of subspaces with $\cap_{n \geq 0} V_{D}^{n}=(0)$ and thus,

$$
H\left(k_{\alpha} \otimes k_{\beta}\right)=\bigoplus_{n \geq 0} V_{D}^{n} \ominus V_{D}^{n+1} .
$$

Our aim is to decompose the space $V_{D}^{n} \ominus V_{D}^{n+1}$ as a direct sum of smaller subspaces and then identify each of these smaller spaces with a space of holomorphic functions on $\mathrm{B}_{d}$. We will work out the details for the case $d=2$.

If we were to follow the analysis used in Section 3 to obtain an orthogonal direct sum decomposition of $V_{D}^{n} \ominus V_{D}^{n+1}$ we would, for each bi-index $N=$ $\left(n_{1}, n_{2}\right)$ with $n_{i} \geq 0, n_{1}+n_{2}=n$, introduce a differential operator of the sort given in (3.2). However we would find that, unlike the case for the polydisk, here the various spaces $\left(\left.\operatorname{Ker} r_{N}^{(0)}\right|_{H\left(k_{\alpha} \otimes k_{\beta}\right)}\right)^{\perp}$ with $|N|=n$ are not mutually orthogonal. Hence we must follow a slightly more intricate path.

Let $r: \operatorname{Hol}\left(\mathrm{B}_{2} \times \mathrm{B}_{2}\right) \rightarrow \operatorname{Hol}\left(\mathrm{B}_{2}\right)$ be the restriction operator

$$
r f(\lambda)=f(\lambda, \lambda)
$$

Again we introduce an appropriate generalization of the transvectants of [9]. For $s>0, N=\left(n_{1}, n_{2}\right)$ with $n_{i} \geq 0, n_{1}+n_{2}=n$ we define the partial differential operator $\mathscr{J}_{N}^{(s)}$ on $\mathrm{Hol}\left(\mathrm{B}_{2} \times \mathrm{B}_{2}\right)$ by

$$
\mathscr{J}_{N}^{(s)}=\sum_{I+J=N} \frac{(-1)^{|J|} N !}{I ! J !(\alpha+s)_{|I|}(\beta+s)_{|J|}}\left(\frac{\partial}{\partial z}\right)^{I}\left(\frac{\partial}{\partial w}\right)^{J} .
$$

Set

$$
r_{N}^{(s)}=r \mathscr{J}_{N}^{(s)} .
$$

We also need the differential operator $\Omega$ defined on $\operatorname{Hol}\left(B_{2} \times B_{2}\right)$ by

$$
\Omega=\frac{\partial}{\partial z_{1}} \frac{\partial}{\partial w_{2}}-\frac{\partial}{\partial z_{2}} \frac{\partial}{\partial w_{1}} .
$$


We now come to the definition of the $n^{\text {th }}$ order restriction operators, $R_{j}^{(n)}$, $j=0, \ldots, n$. First, for $j=0, \ldots, n$ we define $L_{j}^{(n)}$ by, for $\lambda$ in $\mathrm{B}_{2}$,

$$
\left(L_{j}^{(n)} f\right)(\lambda)=\sum_{|N|=n-j} \frac{(n-j) !}{N !} \lambda^{N}\left(r_{N}^{(j)} f\right)(\lambda)
$$

and then define $R_{j}^{(n)}: \operatorname{Hol}\left(\mathrm{B}_{2} \times \mathrm{B}_{2}\right) \rightarrow \operatorname{Hol}\left(\mathrm{B}_{2}\right)$ by

$$
R_{j}^{(n)}=\frac{1}{(\alpha)_{j}(\beta)_{j}} L_{j}^{(n)} \Omega^{j} .
$$

We will use the same notation, $R_{j}^{(n)}$, to denote the restriction of this operator to the space $H\left(k_{\alpha} \otimes k_{\beta}\right)$. We will show that for each $n \geq 0$ the spaces

$$
\left(\operatorname{Ker} R_{j}^{(n)}\right)^{\perp}, \quad j=0, \ldots, n
$$

are mutually orthogonal and together span $V_{D}^{n} \ominus V_{D}^{n+1}$. We will then identify each of these spaces with a weighted Bergman space on $B_{2}$.

At this point, for typographic convenience, we introduce the abbreviation $\Delta_{12}:=z_{1} w_{2}-z_{2} w_{1}$.

To determine the null space of $R_{j}^{(n)}$ we first compute the action of $R_{j}^{(n)}$ on the kernel functions $k_{\alpha}(\cdot, \zeta) \otimes k_{\beta}(\cdot, \eta), \zeta, \eta \in \mathrm{B}_{2}$. Fix $0 \leq j \leq n$. We have, for $\zeta, \eta \in \mathrm{B}_{2}$,

$$
\Omega^{j} k_{\alpha}(\cdot, \zeta) \otimes k_{\beta}(\cdot, \eta)=(\alpha)_{j}(\beta)_{j}\left(\overline{\zeta_{1} \eta_{2}-\zeta_{2} \eta_{1}}\right)^{j} k_{\alpha+j}(\cdot, \zeta) \otimes k_{\beta+j}(\cdot, \eta)
$$

and for $N \in \mathrm{Z}_{+}^{2}$ with $|N|=n-j$,

$$
\begin{aligned}
& \left(r_{N}^{(j)} k_{\alpha+j}(\cdot, \zeta) \otimes k_{\beta+j}(\cdot, \eta)\right)(\lambda) \\
& \quad=\left(\frac{\bar{\zeta}}{1-\langle\lambda, \zeta\rangle_{2}}-\frac{\bar{\eta}}{1-\langle\lambda, \eta\rangle_{2}}\right)^{N} k_{\alpha+j}(\lambda, \zeta) k_{\beta+j}(\lambda, \eta) \\
& \quad=\left(\bar{\zeta}-\bar{\eta}-\left(\overline{\zeta_{1} \eta_{2}-\zeta_{2} \eta_{1}}\right) \underline{\lambda}\right)^{N} k_{\alpha+n}(\lambda, \zeta) k_{\beta+n}(\lambda, \eta)
\end{aligned}
$$

where for $\lambda \in \operatorname{Hol}\left(B_{2}\right), \underline{\lambda}=\left(\lambda_{2},-\lambda_{1}\right)$. Since $\langle\lambda, \underline{\lambda}\rangle_{2}=0$ for all $\lambda \in B_{2}$,

$$
\left(L_{j}^{(n)} k_{\alpha+j}(\cdot, \zeta) k_{\beta+j}(\cdot, \eta)\right)(\lambda)=\langle\lambda, \zeta-\eta\rangle_{2}^{n-j} k_{\alpha+n}(\lambda, \zeta) k_{\beta+n}(\lambda, \eta) .
$$

Thus for all $\zeta, \eta, \lambda \in \mathrm{B}_{2}$,

$$
\begin{aligned}
\left(R_{j}^{(n)} k_{\alpha}(\cdot, \zeta) \otimes k_{\beta}(\cdot, \eta)\right)(\lambda) & \\
& =\left(\overline{\zeta_{1} \eta_{2}-\zeta_{2} \eta_{1}}\right)^{j}\langle\lambda, \zeta-\eta\rangle_{2}^{n-j} k_{\alpha+n}(\lambda, \zeta) k_{\beta+n}(\lambda, \eta) .
\end{aligned}
$$


On the other hand, the reproducing property yields

$$
\begin{aligned}
\left(R_{j}^{(n)}\right. & \left.k_{\alpha}(\cdot, \zeta) \otimes k_{\beta}(\cdot, \eta)\right)(\lambda) \\
& =\sum_{|N|=n-j} \frac{(n-j) !}{N !} \lambda^{N}\left\langle\mathscr{J}_{N}^{(j)} \Omega^{j} k_{\alpha}(\cdot, \zeta) \otimes k_{\beta}(\cdot, \eta), k_{\alpha}(\cdot, \lambda) \otimes k_{\beta}(\cdot, \lambda)\right\rangle \\
& =\left\langle k_{\alpha}(\cdot, \zeta) \otimes k_{\beta}(\cdot, \eta), \sum_{|N|=n-j} \frac{(n-j) !}{N !} \bar{\lambda}^{N} \mathscr{J}_{N}^{(j)^{*}} \Omega^{j^{*}} k_{\alpha}(\cdot, \lambda) \otimes k_{\beta}(\cdot, \lambda)\right\rangle .
\end{aligned}
$$

It follows that for each $\lambda \in \mathrm{B}_{2}$,

$\sum_{|N|=n-j} \frac{(n-j) !}{N !} \bar{\lambda}^{N} \mathscr{J}_{N}^{(j)^{*}} \Omega^{j^{*}}\left(k_{\alpha}(\cdot, \lambda) \otimes k_{\beta}(\cdot, \lambda)\right)=\left(\Delta_{12}\right)^{j}\langle z-w, \lambda\rangle_{2}^{n-j} \eta_{\lambda}^{(n)}$

where

$$
\eta_{\lambda}^{(n)}(z, w)=k_{\alpha+n}(z, \lambda) k_{\beta+n}(w, \lambda) .
$$

Now let $f \in H\left(k_{\alpha} \otimes k_{\beta}\right)$ be in the domain of $R_{j}^{(n)}$ with $R_{j}^{(n)} f=0$. Then for all $\lambda \in \mathrm{B}_{2}$,

$$
\begin{aligned}
0 & =\left(R_{j}^{(n)} f\right)(\lambda)=\sum_{|N|=n-j} \frac{(n-j) !}{N !} \lambda^{N}\left\langle\mathscr{F}_{N}^{(j)} \Omega^{j} f, k_{\alpha}(\cdot, \lambda) \otimes k_{\beta}(\cdot, \lambda)\right\rangle \\
& =\left\langle f,\left(\Delta_{12}\right)^{j}\langle z-w, \lambda\rangle_{2}^{n-j} k_{\alpha+n}(z, \lambda) k_{\beta+n}(w, \lambda)\right\rangle .
\end{aligned}
$$

Thus,

$$
\left(\operatorname{Ker} R_{j}^{(n)}\right)^{\perp}=\bigvee_{\lambda \in \mathrm{B}_{2}}\left\{\left(\Delta_{12}\right)^{j}\langle z-w, \lambda\rangle_{2}^{n-j} k_{\alpha+n}(z, \lambda) k_{\beta+n}(w, \lambda)\right\} .
$$

Clearly $\left(\operatorname{Ker} R_{j}^{(n)}\right)^{\perp} \subseteq V_{D}^{n}$ for all $n \geq 0$ and $j=0, \ldots, n$. Because $R_{j}^{(n)} f=0$ for all $f \in V_{D}^{n+1},\left(\operatorname{Ker} R_{j}^{(n)}\right)^{\perp} \subseteq V_{D}^{n+1^{\perp}}$. Hence, for $n \geq 0$

$$
\bigvee_{j=0, \ldots, n}\left(\operatorname{Ker} R_{j}^{(n)}\right)^{\perp} \subseteq V_{D}^{n} \ominus V_{D}^{n \perp} .
$$

We now establish that for $0 \leq i, j \leq n, i \neq j$

$$
R_{j}^{(n)}\left(\operatorname{Ker} R_{i}^{(n)}\right)^{\perp}=(0)
$$

and determine a formula for

$$
R_{j}^{(n)}\left(\left(\Delta_{12}\right)^{j}\langle z-w, \lambda\rangle_{2}^{n-j} k_{\alpha+n}(z, \lambda) k_{\beta+n}(w, \lambda)\right) .
$$


Define

$$
\begin{aligned}
\mathscr{R} & :=z_{1} \frac{\partial}{\partial z_{1}}+z_{2} \frac{\partial}{\partial z_{2}}+w_{1} \frac{\partial}{\partial w_{1}}+w_{2} \frac{\partial}{\partial w_{2}} \\
(\mathscr{R}+m)_{l} & :=(\mathscr{R}+m)(\mathscr{R}+m+1) \cdots(\mathscr{R}+m+l-1), \quad m, l \in \mathbf{Z}_{+} \\
D_{i} & :=\frac{\partial}{\partial z_{i}}+\frac{\partial}{\partial w_{i}}, \quad i=1,2 \\
N(\lambda) & :=\lambda_{1} D_{1}+\lambda_{2} D_{2}, \quad \lambda \in \mathrm{B}_{2} \\
\hat{\lambda} & :=\left(\overline{\lambda_{2}},-\overline{\lambda_{1}}\right), \quad \lambda \in \mathrm{B}_{2} .
\end{aligned}
$$

It is easy to check that $\Omega \mathscr{R}=(\mathscr{R}+2) \Omega$ and $N(\lambda) \mathscr{R}=(\mathscr{R}+1) N(\lambda)$ for all $\lambda \in \mathrm{B}_{2}$. We will use the following identities each of which can be easily verified by direct computation. For all integers $i \geq 0$ and for all $\lambda \in \mathrm{B}_{2}$,

$$
\begin{gathered}
\Omega\left(\Delta_{12}\right)^{i}=\left(\Delta_{12}\right)^{i} \Omega+i\left(\Delta_{12}\right)^{i-1}(\mathscr{R}+i+1) \\
\Omega\langle z-w, \lambda\rangle_{2}^{i}=\langle z-w, \lambda\rangle_{2}^{i} \Omega-i\langle z-w, \lambda\rangle_{2}^{i-1} N(\hat{\lambda}) .
\end{gathered}
$$

We note in passing that the first of these identities, when applied to the constant function, yields the $n=2$ case of the classical Cayley identity which states that for an $n \times n$ matrix $\left(z_{i j}\right)$ one has

$$
\operatorname{det}\left(\partial_{i j}\right) \operatorname{det}\left(z_{i j}\right)^{m}=(m)_{n} \operatorname{det}\left(z_{i j}\right)^{m-1}
$$

Proposition 4.1. Let $\lambda \in \mathrm{B}_{2}$. For $0 \leq j \leq i \leq n$

$$
\begin{aligned}
& \Omega^{j}\left(\Delta_{12}\right)^{i}\langle z-w, \lambda\rangle_{2}^{n-i} k_{\alpha+n}(\cdot, \lambda) \otimes k_{\beta+n}(\cdot, \lambda) \\
& \quad=j !\left(\begin{array}{l}
i \\
j
\end{array}\right)\left(\Delta_{12}\right)^{i-j}\langle z-w, \lambda\rangle_{2}^{n-i}(\mathscr{R}+2+n-j)_{j} k_{\alpha+n}(\cdot, \lambda) \otimes k_{\beta+n}(\cdot, \lambda)
\end{aligned}
$$

If $0 \leq i<j \leq n$ then

$$
\Omega^{j}\left(\Delta_{12}\right)^{i}\langle z-w, \lambda\rangle_{2}^{n-i} k_{\alpha+n}(\cdot, \lambda) \otimes k_{\beta+n}(\cdot, \lambda)=0 .
$$

Proof. Set $\eta_{\lambda}^{(n)}=k_{\alpha+n}(\cdot, \lambda) \otimes k_{\beta+n}(\cdot, \lambda)$. Note that $\Omega \eta_{\lambda}^{(n)}=N(\hat{\lambda}) \eta_{\lambda}^{(n)}=$ 0 . By the identity (4.11)

$$
\Omega\langle z-w, \lambda\rangle_{2}^{l} \eta_{\lambda}^{(n)}=\langle z-w, \lambda\rangle_{2}^{l} \Omega \eta_{\lambda}^{(n)}-l\langle z-w, \lambda\rangle_{2}^{l-1} N(\hat{\lambda}) \eta_{\lambda}^{(n)}=0
$$


for all $l \geq 0$. If $i \geq 1$ then by (4.11),

$$
\begin{aligned}
& \Omega\left(\Delta_{12}\right)^{i}\langle z-w, \lambda\rangle_{2}^{n-i} \eta_{\lambda}^{(n)} \\
& \quad=\left(\Delta_{12}\right)^{i} \Omega\langle z-w, \lambda\rangle_{2}^{n-i} \eta_{\lambda}^{(n)}+i\left(\Delta_{12}\right)^{i-1}(\mathscr{R}+i+1)\langle z-w, \lambda\rangle_{2}^{n-i} \eta_{\lambda}^{(n)} \\
& \quad=i\left(\Delta_{12}\right)^{i-1}(\mathscr{R}+i+1)\langle z-w, \lambda\rangle_{2}^{n-i} \eta_{\lambda}^{(n)} .
\end{aligned}
$$

Because $\Omega \mathscr{R}=(\mathscr{R}+2) \Omega$, we have for any polynomial $p(x)$ that $\Omega p(\mathscr{R}) \eta_{\lambda}^{(n)}=$ $p(\mathscr{R}+2) \Omega \eta_{\lambda}^{(n)}=0$. Similarly for all polynomials $p(x), N(\hat{\lambda}) p(\mathscr{R}) \eta_{\lambda}^{(n)}=$ $p(\mathscr{R}+1) N(\hat{\lambda}) \eta_{\lambda}^{(n)}=0$. Thus for all $l \geq 0$ and for all polynomials $p(x)$,

$$
\begin{aligned}
\Omega p(\mathscr{R}) & \langle z-w, \lambda\rangle_{2}^{l} \eta_{\lambda}^{(n)} \\
& =p(\mathscr{R}+2) \Omega\langle z-w, \lambda\rangle_{2}^{l} \eta_{\lambda}^{(n)} \\
& =p(\mathscr{R}+2)\left(\langle z-w, \lambda\rangle_{2}^{l} \Omega \eta_{\lambda}^{(n)}-l\langle z-w, \lambda\rangle_{2}^{l-1} N(\hat{\lambda}) \eta_{\lambda}^{(n)}\right) \\
& =0 .
\end{aligned}
$$

This observation together with a repetition of the argument above shows that for $0 \leq j \leq i \leq n$

$$
\begin{aligned}
\Omega^{j}\left(\Delta_{12}\right)^{i}\langle z-w, \lambda\rangle_{2}^{n-i} \eta_{\lambda}^{(n)} & \\
& =i(i-1) \ldots(i-j+1)\left(\Delta_{12}\right)^{i-j} p_{i, j}(\mathscr{R})\langle z-w, \lambda\rangle_{2}^{n-i} \eta_{\lambda}^{(n)}
\end{aligned}
$$

where $p_{i, j}(x)=(x+i+1)(x+i) \ldots(x+i-j+2)$. Since $\mathscr{R}\langle z-w, \lambda\rangle_{2}^{n-i} \eta_{\lambda}^{(n)}=$ $\langle z-w, \lambda\rangle_{2}^{n-i}(\mathscr{R}+n-i) \eta_{\lambda}^{(n)}$ it follows that

$$
p_{i, j}(\mathscr{R})\langle z-w, \lambda\rangle_{2}^{n-i} \eta_{\lambda}^{(n)}=\langle z-w, \lambda\rangle_{2}^{n-i}(\mathscr{R}+2+n-j)_{j} \eta_{\lambda}^{(n)} .
$$

Thus, for $0 \leq j \leq i \leq n$,

$$
\begin{aligned}
& \Omega^{j}\left(\Delta_{12}\right)^{i}\langle z-w, \lambda\rangle_{2}^{n-i} \eta_{\lambda}^{(n)} \\
& \quad=i(i-1) \ldots(i-j+1)\left(\Delta_{12}\right)^{i-j}\langle z-w, \lambda\rangle_{2}^{n-i}(\mathscr{R}+2+n-j)_{j} \eta_{\lambda}^{(n)} .
\end{aligned}
$$

In particular,

$$
\Omega^{i}\left(\Delta_{12}\right)^{i}\langle z-w, \lambda\rangle_{2}^{n-i} \eta_{\lambda}^{(n)}=i !\langle z-w, \lambda\rangle_{2}^{n-i}(\mathscr{R}+2+n-i)_{i} \eta_{\lambda}^{(n)} .
$$

If $0 \leq i<j \leq n$ then

$$
\begin{aligned}
\Omega^{j}\left(\Delta_{12}\right)^{i}\langle z-w, \lambda\rangle_{2}^{n-i} \eta_{\lambda}^{(n)} & =\Omega^{j-i}\left(i !\langle z-w, \lambda\rangle_{2}^{n-i}(\mathscr{R}+2+n-i)_{i} \eta_{\lambda}^{(n)}\right) \\
& =0
\end{aligned}
$$


Suppose now that $l+m=n-j$ and that $f \in \operatorname{Hol}\left(\mathrm{B}_{2} \times \mathrm{B}_{2}\right)$. We will determine a form for $L_{j}^{(n)}\left(\Delta_{12}\right)^{l}\langle z-w, \lambda\rangle_{2}^{m} f$. The proof used for Proposition 3.1 can also be used to show that for multi-indices $M, N \in \mathrm{Z}_{+}^{2}$ with $|M|=|N|$

$$
r_{N}^{(s)}(z-w)^{M}= \begin{cases}\left\|(z-w)^{N}\right\|_{(\alpha+s) \otimes(\beta+s)}^{2} r & M=N \\ 0 & M \neq N .\end{cases}
$$

Furthermore, a quick computation reveals that the norm of $\frac{1}{N !}(z-w)^{N}$ in the space $H\left(k_{\alpha+s} \otimes k_{\beta+s}\right)$ depends only on the length of $N$ and the parameters $\alpha$, $\beta$ and $s$. In particular, if $|N|=n$ then, for $s>0$

$$
\left\|(z-w)^{N}\right\|_{(\alpha+s) \otimes(\beta+s)}^{2}=N ! \sum_{i=0}^{n}\left(\begin{array}{l}
n \\
i
\end{array}\right) \frac{1}{(\alpha+s)_{i}(\beta+s)_{n-i}} .
$$

Consequently, if $f(z, w)=\sum_{|N|=n-j}(z-w)^{N} f_{N}(z, w) \in \operatorname{Hol}\left(\mathrm{B}_{2} \times \mathrm{B}_{2}\right)$ then, for $\lambda \in \mathrm{B}_{2}$

$$
\left(L_{j}^{(n)} f\right)(\lambda)=C_{j}^{(n)} \sum_{|N|=n-j} \lambda^{N} f_{N}(\lambda, \lambda)
$$

where

$$
C_{j}^{(n)}=(n-j) ! \sum_{i=0}^{n-j}\left(\begin{array}{c}
n-j \\
i
\end{array}\right) \frac{1}{(\alpha+j)_{i}(\beta+j)_{n-j-i}} .
$$

Proposition 4.2. Let $\lambda \in \mathrm{B}_{2}$ and $0 \leq j \leq n$. If $m, l \geq 0$ with $m+l=n-j$ then for all $f \in \operatorname{Hol}\left(B_{2} \times B_{2}\right)$

$$
\left(L_{j}^{(n)}\left(\Delta_{12}\right)^{l}\langle z-w, \lambda\rangle_{2}^{m} f\right)(\zeta)= \begin{cases}0 & l \neq 0 \\ C_{j}^{(n)}\langle\zeta, \lambda\rangle_{2}^{n-j} f(\zeta, \zeta) & l=0 .\end{cases}
$$

Proof. Set $\underline{z}=\frac{1}{2}\left(z_{2},-z_{1}\right)$. Then, recalling (1.1),

$$
\begin{aligned}
\left(\Delta_{12}\right)^{i} & =\left(\left(z_{1}-w_{1}\right)\left(\frac{z_{2}+w_{2}}{2}\right)-\left(z_{2}-w_{2}\right)\left(\frac{z_{1}+w_{1}}{2}\right)\right)^{i} \\
& =\sum_{|I|=i} \frac{i !}{I !}(z-w)^{I}(\underline{z+w})^{I} .
\end{aligned}
$$

Hence,

$$
\left(\Delta_{12}\right)^{l}\langle z-w, \lambda\rangle_{2}^{m}=\sum_{|L|=l} \sum_{|M|=m} \frac{l ! m !}{L ! M !}(z-w)^{L+M}(\underline{z+w})^{L} \bar{\lambda}^{M} .
$$


By (4.13),

$$
\begin{aligned}
\left(L_{j}^{(n)}\left(\Delta_{12}\right)^{l}\langle z-w, \lambda\rangle_{2}^{m} f\right)(\zeta) & =C_{j}^{(n)} \sum_{|L|=l} \sum_{|M|=m} \frac{l ! m !}{L ! M !} \zeta^{L+M}(\underline{\zeta})^{L} \bar{\lambda}^{M} f(\zeta, \zeta) \\
& =C_{j}^{(n)}\langle\zeta, \underline{\zeta}\rangle_{2}^{l}\langle\zeta, \lambda\rangle_{2}^{m} f(\zeta, \zeta) .
\end{aligned}
$$

Because $\langle\zeta, \bar{\zeta}\rangle_{2}=0$ for all $\zeta \in \mathrm{B}_{2}$, the result follows.

Note that the restriction map $r: \operatorname{Hol}\left(B_{2} \times B_{2}\right) \rightarrow \operatorname{Hol}\left(B_{2}\right)$ defined by $(r f)(\lambda)=f(\lambda, \lambda)$ intertwines the radial derivative in four variables and the radial derivative in two variables. In our notation: $r \mathscr{R}=\mathscr{R}_{2} r$ where $\mathscr{R}_{2}=$ $\lambda_{1} \frac{\partial}{\partial \lambda_{1}}+\lambda_{2} \frac{\partial}{\partial \lambda_{2}}$. Hence by Propositions 4.1 and 4.2 we arrive at the following.

Proposition 4.3. Fix $0 \leq i, j \leq n$. Set $\eta_{\lambda}^{(n)}(z, w)=k_{\alpha+n}(z, \lambda) k_{\beta}(w, \lambda)$, $\lambda \in \mathbf{B}_{2}$. If $i \neq j$ then $R_{j}^{(n)}\left(\Delta_{12}\right)^{i}\langle z-w, \lambda\rangle_{2}^{n-i} \eta_{\lambda}^{(n)}=0$ for all $\lambda \in \mathbf{B}_{2}$. For $i=j$ we have the following formula, valid for all $\lambda \in \mathrm{B}_{2}$.

$$
\begin{aligned}
R_{j}^{(n)}\left(\Delta_{12}\right)^{j}\langle z-w, \lambda\rangle_{2}^{n-j} \eta_{\lambda}^{(n)} \\
=\frac{j ! C_{j}^{(n)}}{(\alpha)_{j}(\beta)_{j}}\langle\cdot, \lambda\rangle_{2}^{n-j}\left(\mathscr{R}_{2}+n+2-j\right)_{j} k_{\alpha+\beta+2 n}(\cdot, \lambda) .
\end{aligned}
$$

Thus by the proposition above, $R_{j}^{(n)}\left(\operatorname{Ker} R_{i}^{(n)}\right)^{\perp}=(0)$ for all $0 \leq i, j \leq$ $n$ with $i \neq j$. Hence the spaces $\left(\operatorname{Ker} R_{j}^{(n)}\right)^{\perp}, j=0, \ldots, n$, are mutually orthogonal. So by (4.9),

$$
\bigoplus_{j=0}^{n}\left(\operatorname{Ker} R_{j}^{(n)}\right)^{\perp} \subseteq V_{D}^{n} \ominus V_{D}^{n+1} .
$$

To show the reverse inclusion it suffices to show that if $f \in V_{D}^{n}$ and $R_{j}^{(n)} f=0$ for all $j=0, \ldots, n$, then $f \in V_{D}^{n+1}$. In other words, we need only show that

$$
V_{D}^{n} \cap\left(\cap_{j=0}^{n} \operatorname{Ker} R_{j}^{(n)}\right)=V_{D}^{n+1} .
$$

We first need a lemma.

Lemma 4.4. Let $g_{0}, g_{1}, \ldots, g_{n} \in \operatorname{Hol}\left(\mathrm{B}_{2}\right)$ and suppose that

(i) $\sum_{j=0}^{n} \lambda_{1}^{j} \lambda_{2}^{n-j} g_{j}(\lambda)=0$ for all $\lambda \in \mathrm{B}_{2}$ and

(ii) $(j+1) \frac{\partial g_{j+1}}{\partial \lambda_{2}}=(n-j) \frac{\partial g_{j}}{\partial \lambda_{1}}$ for $j=0, \ldots, n-1$.

Then $g_{j}=0$ for $j=0, \ldots, n$. 
Proof. The proof will be by induction. For $n=1$, if both (i) and (ii) above hold then differentiating, with respect to $\lambda_{1}$, both sides of the equation in (i) yields $\mathscr{R}_{2} g_{1}=-g_{1}$. Hence, $g_{1}=0$ and so by (i), $g_{0}=0$. Suppose now that the result holds for some $n \geq 1$. Let $g_{0}, g_{1}, \ldots, g_{n+1} \in \operatorname{Hol}\left(\mathrm{B}_{2}\right)$ satisfy

(a) $\sum_{j=0}^{n+1} \lambda_{1}^{j} \lambda_{2}^{n+1-j} g_{j}(\lambda)=0$ for all $\lambda \in \mathrm{B}_{2}$ and

(b) $(j+1) \frac{\partial g_{j+1}}{\partial \lambda_{2}}=(n+1-j) \frac{\partial g_{j}}{\partial \lambda_{1}}$ for $j=0, \ldots, n$.

Set $h_{j}=\left(\frac{j+1}{n+1}\right) g_{j+1}, j=0, \ldots, n$. We will show that the functions $\left\{h_{j}\right\}_{j=0}^{n}$ satisfy both (i) and (ii). By condition (a), $\frac{\partial}{\partial \lambda_{1}}\left(\sum_{j=0}^{n+1} \lambda_{1}^{j} \lambda_{2}^{n+1-j} g_{j}(\lambda)\right)=0$ and from condition (b) it easily follows that

$$
\frac{\partial}{\partial \lambda_{1}}\left(\sum_{j=0}^{n+1} \lambda_{1}^{j} \lambda_{2}^{n+1-j} g_{j}(\lambda)\right)=\left(\mathscr{R}_{2}+I\right)\left(\sum_{j=0}^{n} \lambda_{1}^{j} \lambda_{2}^{n-j} h_{j}(\lambda)\right) .
$$

Thus, $\sum_{j=0}^{n} \lambda_{1}^{j} \lambda_{2}^{n-j} h_{j}(\lambda)=0$ for all $\lambda \in \mathrm{B}_{2}$. For $j=0, \ldots, n-1$,

$$
\begin{aligned}
(j+1) \frac{\partial h_{j+1}}{\partial \lambda_{2}} & =\left(\frac{j+1}{n+1}\right)(j+2) \frac{\partial g_{j+2}}{\partial \lambda_{2}} \\
& =\left(\frac{j+1}{n+1}\right)(n+1-j-1) \frac{\partial g_{j+1}}{\partial \lambda_{1}} \\
& =(n-j) \frac{\partial h_{j}}{\partial \lambda_{1}} .
\end{aligned}
$$

Thus, by assumption, $h_{j}=0, j=0, \ldots, n$ and so $g_{j}=0, j=1, \ldots, n+1$. Condition (a) now implies that $g_{0}=0$.

Proposition 4.5. Let $f_{0}, \ldots, f_{n} \in \operatorname{Hol}\left(\mathrm{B}_{2} \times \mathrm{B}_{2}\right)$. Set

$$
f(z, w)=\sum_{i=0}^{n}\left(z_{1}-w_{1}\right)^{i}\left(z_{2}-w_{2}\right)^{n-i} f_{i}(z, w) .
$$

If $R_{j}^{(n)} f=0, j=0, \ldots, n$ then $r f_{i}=0, i=0, \ldots, n$.

Proof. First note that

$$
\begin{aligned}
& (\Omega f)(z, w)=\sum_{i=0}^{n}\left(z_{1}-w_{1}\right)^{i}\left(z_{2}-w_{2}\right)^{n-i}\left(\Omega f_{i}\right)(z, w) \\
& +\sum_{i=0}^{n-1}\left(z_{1}-w_{1}\right)^{i}\left(z_{2}-w_{2}\right)^{n-1-i}\left((i+1)\left(D_{2} f_{i+1}\right)(z, w)-(n-i)\left(D_{1} f_{i}\right)(z, w)\right) .
\end{aligned}
$$


For $j=0, \ldots, n$ define the functions $\left\{h_{i}^{(j)}\right\}_{i=0}^{n-j}$ by $h_{i}^{(0)}=f_{i}, i=0, \ldots, n$, and for $j \geq 1, i=0, \ldots, n-j$

$$
h_{i}^{(j)}=(i+1) D_{2} h_{i+1}^{(j-1)}-(n-(j-1)-i) D_{1} h_{i}^{(j-1)} .
$$

From the formula for $\Omega f$ we see that the functions $\left\{h_{i}^{(j)}\right\}_{i=0}^{n-j}$ are defined to satisfy, for $j=0, \ldots, n$,

$$
\Omega^{j} f-\sum_{i=0}^{n-j}\left(z_{1}-w_{1}\right)^{i}\left(z_{2}-w_{2}\right)^{n-j-i} h_{i}^{(j)} \in V_{D}^{n-j+1} .
$$

Since $L_{j}^{(n)} V_{D}^{n-j+1}=(0)$ for $j=0, \ldots, n$ we have by (4.13)

$$
\begin{aligned}
\left(L_{j}^{(n)} \Omega^{j} f\right)(\lambda) & =L_{j}^{(n)}\left(\sum_{i=0}^{n-j}\left(z_{1}-w_{1}\right)^{i}\left(z_{2}-w_{2}\right)^{n-j-i} h_{i}^{(j)}\right)(\lambda) \\
& =C_{j}^{(n)} \sum_{i=0}^{n-j} \lambda_{1}^{i} \lambda_{2}^{n-j-i} h_{i}^{(j)}(\lambda, \lambda)
\end{aligned}
$$

for all $\lambda \in \mathrm{B}_{2}$. By assumption $R_{j}^{(n)} f=0, j=0, \ldots, n$, and thus, for all $\lambda \in \mathrm{B}_{2}$ and for $j=0, \ldots, n$,

$$
\sum_{i=0}^{n-j} \lambda_{1}^{i} \lambda_{2}^{n-j-i} h_{i}^{(j)}(\lambda, \lambda)=0 .
$$

For $j=0, \ldots, n$ define the functions $\left\{g_{i}^{(j)}\right\}_{i=0}^{n-j}$ in $\operatorname{Hol}\left(\mathrm{B}_{2}\right)$ by, for $\lambda \in \mathrm{B}_{2}$, $g_{i}^{(j)}(\lambda)=h_{i}^{(j)}(\lambda, \lambda)$. In particular, $g_{i}^{(0)}=r f_{i}, i=0, \ldots, n$ and, since $r D_{i}=$ $\frac{\partial}{\partial \lambda_{i}} r, i=1,2$, for $j \geq 1$ and $i=0, \ldots, n-j$, we have

$$
g_{i}^{(j)}=(i+1) \frac{\partial g_{i+1}^{(j-1)}}{\partial \lambda_{2}}-(n-(j-1)-i) \frac{\partial g_{i}^{(j-1)}}{\partial \lambda_{1}} .
$$

Also, by (4.14), we have that for $\lambda$ in $\mathrm{B}_{2}, j=0, \ldots, n$,

$$
\sum_{i=0}^{n-j} \lambda_{1}^{i} \lambda_{2}^{n-j-i} g_{i}^{(j)}(\lambda)=0 .
$$

In particular, $g_{0}^{(n)}=0$ which implies by (4.15) (with $j=n$ ) that

$$
\frac{\partial g_{1}^{(n-1)}}{\partial \lambda_{2}}=\frac{\partial g_{0}^{(n-1)}}{\partial \lambda_{1}} \text {. }
$$


By (4.16) with $(j=n-1), \lambda_{2} g_{0}^{(n-1)}(\lambda)+\lambda_{1} g_{1}^{(n-1)}(\lambda)=0$, for all $\lambda \in \mathrm{B}_{2}$. It follows by Lemma 4.4 that $g_{0}^{(n-1)}=g_{1}^{(n-1)}=0$. This in turn implies, by (4.15) (with $j=n-1$ ), that for $i=0,1,2$

$$
(i+1) \frac{\partial g_{i+1}^{(n-2)}}{\partial \lambda_{2}}=(2-i) \frac{\partial g_{i}^{(n-2)}}{\partial \lambda_{1}} \text {. }
$$

Again, by (4.16) with ( $j=n-2), \sum_{i=0}^{2} \lambda_{1}^{i} \lambda_{2}^{2-i} g_{i}(\lambda)=0$ for all $\lambda \in \mathrm{B}_{2}$. Thus, by Lemma 4.4, $g_{i}^{(n-2)}=0, i=0,1,2$. Continuing in this fashion we arrive at $g_{i}^{(0)}=r f_{i}=0, i=0, \ldots, n$. The proof is now complete.

CoRollary 4.6. $V_{D}^{n} \ominus V_{D}^{n+1}=\bigoplus_{j=0}^{n}\left(\operatorname{Ker} R_{j}^{(n)}\right)^{\perp}, n \geq 0$.

Now we want to identify the relative quotient $V_{D}^{n} \ominus V_{D}^{n+1}$ with a direct sum of weighted Bergman spaces on $\mathrm{B}_{2}$. To be precise, for each $j=0, \ldots, n$, we will identify the space $\left(\operatorname{Ker} R_{j}^{(n)}\right)^{\perp}$ with the subspace of functions in $H\left(k_{\alpha+\beta+2 n+j}\right)$ which vanish up to order $n-j$ at the origin. To do this, define kernel functions $K_{j}^{(n)}, j=0, \ldots, n$, on $\mathrm{B}_{2} \times \mathrm{B}_{2}$ by

$$
K_{j}^{(n)}(z, \lambda)=\langle z, \lambda\rangle_{2}^{n-j} k_{\alpha+\beta+2 n+j}(z, \lambda)=\langle z, \lambda\rangle_{2}^{n-j}\left(\frac{1}{1-\langle z, \lambda\rangle_{2}}\right)^{\alpha+\beta+2 n+j} .
$$

It is easy to see that for each $0 \leq j \leq n$ the space generated by the kernel function $K_{j}^{(n)}$ is

$$
\left\{\sum_{i=0}^{n-j} z_{1}^{i} z_{2}^{n-j-i} f_{i}(z): f_{0}, \ldots, f_{n-j} \in H\left(k_{\alpha+\beta+2 n+j}\right)\right\} .
$$

We provide a quick proof of this for general parameters.

Proposition 4.7. Fix an integer $m \geq 0$ and a parameter $\gamma>0$. Let $k_{\gamma}(z, \lambda)=\left(1-\langle z, \lambda\rangle_{2}\right)^{-\gamma}, z, \lambda \in \mathrm{B}_{2}$. Set $K_{m}(z, \lambda)=\langle z, \lambda\rangle_{2}^{m} k_{\gamma}(z, \lambda), z, \lambda \in$ $\mathrm{B}_{2}$ and let $H\left(K_{m}\right)$ denote the Hilbert space of holomorphic functions on $\mathrm{B}_{2}$ with reproducing kernel $K_{m}$. Then

$$
H\left(K_{m}\right)=\left\{\sum_{i=0}^{m} z_{1}^{i} z_{2}^{m-i} f_{i}(z): f_{0}, \ldots, f_{m} \in H\left(k_{\gamma}\right)\right\}
$$

with norm given by

$$
\|f\|_{H\left(K_{m}\right)}=\inf \sqrt{\sum_{i=0}^{m}\left\|f_{i}\right\|_{H\left(k_{\gamma}\right)}^{2}}
$$


where the infimum is over all $(m+1)$-tuples $\left(f_{0}, \ldots, f_{m}\right) \in \oplus_{i=0}^{m} H\left(k_{\gamma}\right)$ with $f(z)=\sum_{i=0}^{m} z_{1}^{i} z_{2}^{m-i} f_{i}(z)$.

Proof. Let $T_{i}=M_{z_{1}}^{i} M_{z_{2}}^{m-i}, i=0, \ldots, m$ acting on the space $H\left(k_{\gamma}\right)$. The positive operator $P=\sum_{i=0}^{m}\left(\begin{array}{c}m \\ i\end{array}\right) T_{i} T_{i}^{*}$ satisfies, for $z, w \in \mathrm{B}_{2}$

$$
\begin{aligned}
\left\langle P k_{\gamma}(\cdot, w), k_{\gamma}(\cdot, z)\right\rangle & =\sum_{i=0}^{m}\left(\begin{array}{c}
m \\
i
\end{array}\right) z_{1}^{i} z_{2}^{m-i} \bar{w}_{1}^{i} \bar{w}_{2}^{m-i} k_{\gamma}(z, w) \\
\bar{w}_{1}^{i} \bar{w}_{2}^{m-i} & =\langle z, w\rangle_{2}^{m} k_{\gamma}(z, w) .
\end{aligned}
$$

Now factor $P=A A^{*}$ where

$$
A=\left(\sqrt{\left(\begin{array}{c}
m \\
0
\end{array}\right)} T_{0}, \sqrt{\left(\begin{array}{c}
m \\
1
\end{array}\right)} T_{1}, \ldots, \sqrt{\left(\begin{array}{c}
m \\
m
\end{array}\right)} T_{m}\right): \bigoplus_{i=0}^{m} H\left(k_{\gamma}\right) \rightarrow H\left(k_{\gamma}\right) .
$$

It follows that

$$
\left\langle A A^{*} k_{\gamma}(\cdot, w), k_{\gamma}(\cdot, z)\right\rangle=\langle z, w\rangle_{2}^{m} k_{\gamma}(z, w), \quad z, w \in \mathrm{B}_{2} .
$$

Thus, the range space of the row operator $A$ coincides (isometrically) with the space $H\left(K_{m}\right)$. Now the range of $A$ is

$$
\left\{\sum_{i=0}^{m} z_{1}^{i} z_{2}^{m-i} f_{i}(z): f_{0}, \ldots, f_{m} \in H\left(k_{\gamma}\right)\right\}
$$

and the range space norm of $A$ is given by

$$
\|A f\|_{\text {Ran } A}=\inf \|g\|
$$

where the infimum is over all $g \in \bigoplus_{i=0}^{m} H\left(k_{\gamma}\right)$ with $A f=A g$. The proof is now complete.

THeOREM 4.8. Fix $n \geq 0$. The operator $R_{j}^{(n)}$ maps $H\left(k_{\alpha} \otimes k_{\beta}\right)$ onto $H\left(K_{j}^{(n)}\right)$, $j=0, \ldots, n$. Consequently, if we decompose $V_{D}^{n} \ominus V_{D}^{n+1}=\bigoplus_{j=0}^{n}\left(\operatorname{Ker} R_{j}^{(n)}\right)^{\perp}$ then the map $\bigoplus_{j=0}^{n} R_{j}^{(n)}: V_{D}^{n} \ominus V_{D}^{n+1} \rightarrow \bigoplus_{j=0}^{n} H\left(K_{j}^{(n)}\right)$ is invertible.

Proof. Fix $0 \leq j \leq n$. Define the kernel function $G_{j}^{(n)}$ on $\mathrm{B}_{2} \times \mathrm{B}_{2}$ by

$$
G_{j}^{(n)}(\zeta, \lambda):=\left(R_{j}^{(n)}\left(\Delta_{12}\right)^{j}\langle z-w, \lambda\rangle_{2}^{n-j} k_{\alpha+n}(\cdot, \lambda) \otimes k_{\beta+n}(\cdot, \lambda)\right)(\zeta) .
$$

By Proposition 4.3, for all $\zeta, \lambda$ in $B_{2}$

$$
G_{j}^{(n)}(\zeta, \lambda)=\frac{j ! C_{j}^{(n)}}{(\alpha)_{j}(\beta)_{j}}\langle\zeta, \lambda)_{2}^{n-j}\left(\mathscr{R}_{2}+n+2-j\right)_{j} k_{\alpha+\beta+2 n}(\zeta, \lambda) .
$$


We first show that the space generated by the kernel functions $\left(\mathscr{R}_{2}+n+2-\right.$ $j)_{j} k_{\alpha+\beta+2 n}$ coincides with the space generated by $k_{\alpha+\beta+2 n+j}$. Both kernels are diagonal and so we just need to compare the weights. Now

$$
\begin{aligned}
\left(\left(\mathscr{R}_{2}+\right.\right. & \left.2+n-j)_{j} k_{\alpha+\beta+2 n}(\cdot, \lambda)\right)(z) \\
& =\sum_{m=0}^{\infty} \frac{(\alpha+\beta+2 n)_{m}}{m !}\left(\mathscr{R}_{2}+2+n-j\right)_{j}\langle z, \lambda\rangle^{m} \\
& =\sum_{m=0}^{\infty} \frac{(\alpha+\beta+2 n)_{m}}{m !}(m+2+n-j)_{j}\langle z, \lambda\rangle^{m} .
\end{aligned}
$$

Asymptotically, we have by Stirling's formula,

$$
\frac{(\gamma)_{m}(m+2+n-j)_{j}}{m !} \sim m^{\gamma-1-j} \sim \frac{(\gamma+j)_{m}}{m !} .
$$

Setting $\gamma=\alpha+\beta+2 n$ we see that the two kernels are equivalent in the sense of positivity, that is they span the same space, namely $H\left(k_{\alpha+\beta+2 n+j}\right)$, and the norms are equivalent. In other words, the inclusion

$$
H\left(\left(\mathscr{R}_{2}+2+n-j\right)_{j} k_{\alpha+\beta+2 n}\right) \rightarrow H\left(k_{\alpha+\beta+2 n+j}\right)
$$

is bounded above and below. The Schur product of kernels preserves equivalence and so the kernels, $\langle\zeta, \lambda\rangle_{2}^{n-j}\left(\mathscr{R}_{2}+n+2-j\right)_{j} k_{\alpha+\beta+2 n}(\zeta, \lambda)$ and $K_{j}^{(n)}(\zeta, \lambda)=\langle\zeta, \lambda\rangle_{2}^{n-j} k_{\alpha+\beta+2 n+j}(\zeta, \lambda)$ are equivalent. Thus, the space generated by $G_{j}^{(n)}$ coincides with the space generated by the kernel $K_{j}^{(n)}$ and we showed above that

$$
H\left(K_{j}^{(n)}\right)=\left\{\sum_{i=0}^{n-j} z_{1}^{i} z_{2}^{n-j-i} f_{i}(z): f_{0}, \ldots, f_{n-j} \in H\left(k_{\alpha+\beta+2 n+j}\right)\right\} .
$$

Set

$$
\eta_{\lambda}^{(n)}=k_{\alpha+n}(\cdot, \lambda) \otimes k_{\beta+n}(\cdot, \lambda) .
$$

By the definition of the kernel $G_{j}^{(n)}$,

$$
\begin{aligned}
\left\langle R_{j}^{(n)}\left(\Delta_{12}\right)^{j}\langle z-w, \lambda\rangle_{2}^{n-j} \eta_{\lambda}^{(n)},\right. & \left.R_{j}^{(n)}\left(\Delta_{12}\right)^{j}\langle z-w, \zeta\rangle_{2}^{n-j} \eta_{\zeta}^{(n)}\right\rangle_{H\left(G_{j}^{(n)}\right)} \\
& =\left\langle G_{j}^{(n)}(\cdot, \lambda), G_{j}^{(n)}(\cdot, \zeta)\right\rangle_{H\left(G_{j}^{(n)}\right)}=G_{j}^{(n)}(\zeta, \lambda) .
\end{aligned}
$$

On the other hand by (4.7)

$$
\left(\sum_{|N|=n-j} \frac{(n-j) !}{N !} \zeta^{N} \mathscr{J}_{N}^{(j)} \Omega^{j}\right)^{*} k_{\alpha}(\cdot, \zeta) \otimes k_{\beta}(\cdot, \zeta)=\left(\Delta_{12}\right)^{j}\langle z-w, \zeta\rangle_{2}^{n-j} \eta_{\zeta}^{(n)}
$$


and thus,

$$
\begin{aligned}
& \left\langle\left(\Delta_{12}\right)^{j}\langle z-w, \lambda\rangle_{2}^{n-j} \eta_{\lambda}^{(n)},\left(\Delta_{12}\right)^{j}\langle z-w, \zeta\rangle_{2}^{n-j} \eta_{\zeta}^{(n)}\right\rangle \\
& =\left\langle\left(\sum_{|N|=n-j} \frac{(n-j) !}{N !} \zeta^{N} \mathscr{J}_{N}^{(j)} \Omega^{j}\right)\left(\Delta_{12}\right)^{j}\langle z-w, \lambda\rangle_{2}^{n-j} \eta_{\lambda}^{(n)}, k_{\alpha}(\cdot, \zeta) \otimes k_{\beta}(\cdot, \zeta)\right\rangle \\
& =\left(R_{j}^{(n)}\left(\Delta_{12}\right)^{j}\langle z-w, \lambda\rangle_{2}^{n-j} \eta_{\lambda}^{(n)}\right)(\zeta) \\
& =G_{j}^{(n)}(\zeta, \lambda)
\end{aligned}
$$

Hence for all $\zeta, \lambda \in \mathrm{B}_{2}$,

$$
\begin{aligned}
\left\langle R_{j}^{(n)}\left(\Delta_{12}\right)^{j}\langle z-\right. & \left.w, \lambda\rangle_{2}^{n-j} \eta_{\lambda}^{(n)}, R_{j}^{(n)}\left(\Delta_{12}\right)^{j}\langle z-w, \zeta\rangle_{2}^{n-j} \eta_{\zeta}^{(n)}\right\rangle_{H\left(G_{j}^{(n)}\right)} \\
& =\left\langle\left(\Delta_{12}\right)^{j}\langle z-w, \lambda\rangle_{2}^{n-j} \eta_{\lambda}^{(n)},\left(\Delta_{12}\right)^{j}\langle z-w, \zeta\rangle_{2}^{n-j} \eta_{\zeta}^{(n)}\right\rangle .
\end{aligned}
$$

It follows that the operator $R_{j}^{(n)}$ establishes an isometric isomorphism between $\left(\operatorname{Ker} R_{j}^{(n)}\right)^{\perp}$ and $H\left(G_{j}^{(n)}\right)$. Since $H\left(G_{j}^{(n)}\right)=H\left(K_{j}^{(n)}\right)$ and the norms are equivalent, the proof is complete.

\section{Questions}

Finally we mention briefly several areas where we think there is interesting work to be done.

First, as noted in the introduction, higher order Hankel forms can be defined by their interaction with a natural group action or by their commutation properties with multiplication operators. We saw in Corollary 3.4 that for classical spaces of functions on the disk and polydisk these two approaches give the same classes of forms. It is not clear if the analogous identification holds for function spaces on the ball. More specifically, for certain function spaces on the ball (4.1) and Corollary 4.8 together give an orthogonal direct sum decomposition of $H\left(k_{\alpha} \otimes k_{\beta}\right)$. There is another direct sum decomposition one can consider. First identify $H\left(k_{\alpha} \otimes k_{\beta}\right)$ with $H\left(k_{\alpha}\right) \otimes H\left(k_{\beta}\right)$ next note that the group of conformal automorphisms of the ball induces a group action on each factor of this tensor product and hence on the product itself. One can now decompose the product into components invariant under this group action and obtain an orthogonal direct sum decomposition of the product and hence also of $H\left(k_{\alpha} \otimes k_{\beta}\right)$. Part of this program, for the ball and for general bounded symmetric domains, is carried out by Peetre in [12] and [13]. It would be quite interesting to know how those results are related to the one we obtained. In a related direction, in [7] the authors identify the irreducible components of tensor product spaces which are, in our notation, $H\left(k_{\alpha}\right) \otimes \overline{H\left(k_{\alpha}\right)}$. 
Second, one theme of classical invariant theory is the study of certain linear differential and partial differential operators which interact in natural ways with an ambient group action. The differential operators which arise when studying higher order Hankel forms are of this sort. It was noticed in [9] that the differential operators (3.2) were instances of transvectants of the sort first introduced by Clebsch. Here we note that the literature on classical invariant theory, the operator $\Omega$ of (4.4) is an instance of the so-called omega process introduced by Cayley. However, at the moment these are merely observations and we do not know if the ideas and tools from invariant theory can used to study higher order Hankel forms. Certainly the representation theoretic approach of, for instance, [12], [13], [7], and [24] suggest this may be possible. For more on invariant theory we refer to (cf. [11]).

Finally, it would be very interesting to understand the results developed here in the context of the general analysis of Hilbert modules and their quotient modules by Douglas, Misra, and Varughese. In particular, in [5] those authors consider a class of quotient modules for which the modules $W^{n}$ which we discuss in Section 3 are natural and non-trivial examples.

\section{Afterword}

After this manuscript was completed the authors learned of work of Peng and Zhang which has since appeared as [15]. In that very interesting paper the authors find, for the tensor product of any pair of (analytic continuations) of the weighted Bergman spaces on bounded symmetric domains, the decomposition of the tensor product into irreducible summands. They also give formulas for the generalized transvectants which intertwine the product to the summands.

\section{REFERENCES}

1. Arazy, J., A survey of invariant Hilbert spaces of analytic functions on bounded symmetric domains, Multivariable operator theory (Seattle, WA, 1993), Curto, Douglas, Pincus and Salinas, eds. 7-65, Contemp. Math. 185 AMS, Providence, RI, 1995.

2. Clark, D. N., Restrictions of $H^{p}$ functions in the polydisk, Amer. J. Math. 110 (1988), 1119 1152.

3. Cowen, C., and MacCluer, B., Composition Operators on Spaces of Analytic Functions, Stud. Adv. Math. (1995).

4. Douglas, R. G., and Misra, G., Geometric invariants for resolutions of Hilbert modules, Operator Theory: Advances and Applications, 104 (1998), 83-112.

5. Douglas, R. G., Misra, G., and Varughese, C., On quotient modules-the case of arbitrary multiplicity, J. Funct. Anal. 174 (2000), 364-398.

6. Douglas, R. G., and Paulsen, V. I., Hilbert Modules Over Function Algebras, Pitman Res. Notes Math. Ser. 217, (1989).

7. Englis, M., Hille, S. C., Peetre, J., Rosengren, H., and Zhang, G., A new kind of HankelToeplitz type operator connected with the complementary series, Arab J. Math. Sci. 6 (2000), no. 2, 49-80. 
8. Horowitz, C., and Oberlin, D. M., Restriction of $H^{p}$ functions to the diagonal of $U^{n}$, Indiana Univ. Math. J. 24 (1975), 767-772.

9. Janson, S., and Peetre, J., A new generalization of Hankel operators (the case of higher weights), Math. Nachr. 132 (1987), 313-328.

10. Janson, S., and Peetre, J., Paracommutators-boundedness and Schatten-von Neumann properties, Trans. Amer. Math. Soc. 305 (1988), 467-504.

11. Olver, P. J., Classical Invariant Theory, London Math. Soc. Stud. Texts 44 (1999).

12. Peetre, J., Hankel kernels of higher weight for the ball, Nagoya Math. J. 130 (1993), 183-192.

13. Peetre, J., Hankel forms of arbitrary weight over a symmetric domain via the transvectant, Rocky Mountain J. Math. 24 (1994), 1065-1085.

14. Peetre, J., and Rochberg, R., Higher order Hankel forms, in Multivariable operator theory (Seattle, WA, 1993), Curto, Douglas, Pincus and Salinas (eds.), 283-306, Contemp. Math. 185 (1995).

15. Peng, L., and Zhang, G., Tensor products of holomorphic representations and bilinear differential operators, J. Funct. Anal. 210 (2004), 171-192.

16. Rochberg, R., Higher order Hankel forms and commutators, in Holomorphic spaces (Berkeley, CA, 1995), Axler and McCarthy (eds.), 155-178, Math. Sci. Res. Inst. Publ. 33 (1998).

17. Rochberg, R., and Wu, Z., A new characterization of Dirichlet type spaces and applications, Illinois J. Math. 37 (1993), 101-122.

18. Rosengren, H., Multilinear Hankel forms of higher order and orthogonal polynomials, Math. Scand. 82 (1998), 53-88.

19. Rosengren, H., Multivariable orthogonal polynomials and coupling coefficients for discrete series representations, SIAM J. Math. Anal. 30 (1999), 232-272.

20. Rudin, W., Function Theory in Polydisks, W. A. Benjamin, Inc., New York - Amsterdam, 1969.

21. Salinas, N., Products of kernel functions and module tensor products, Oper. Theory Adv. Appl. 32 (1988), 219-241.

22. Wu, Z., A class of bilinear forms on Dirichlet type spaces, J. London Math. Soc. 54 (1996), 498-514.

23. Zhang, G., Ha-plitz operators between Möbius invariant subspaces, Math. Scand. 71 (1992), 69-84.

24. Zhang, G., Tensor products of weighted Bergman spaces and invariant Ha-plitz operators, Math. Scand. 71 (1992), 85-95.

DEPARTMENT OF MATHEMATICS

WAYNE STATE UNIVERSITY

DETROIT, MI 48202

USA

E-mail: sarah@math.wayne.edu
DEPARTMENT OF MATHEMATICS WASHINGTON UNIVERSITY IN ST. LOUIS ST. LOUIS, MO 63130

USA

E-mail: rr@math.wustl.edu 